\title{
A checklist of the non-leaf-cutting fungus-growing ants (Hymenoptera, Formicidae) from Colombia, with new biogeographic records
}

\author{
Daniela Mera-Rodríguez ${ }^{1,2}$, Francisco Serna ${ }^{1}$, Jeffrey Sosa-Calvo ${ }^{2,3}$, John Lattke ${ }^{4}$, \\ Christian Rabeling ${ }^{2}$
}

1 Universidad Nacional de Colombia, Facultad de Ciencias Agrarias, Museo Entomológico UNAB, Grupo Sistemática de Insectos Agronomía (SIA), Carrera 30 \#45-03, Bogotá D.C., 111321, Colombia. 2 School of Life Sciences, Arizona State University, 427 E Tyler Mall, Tempe, AZ, 85281, USA. 3 Department of Entomology, National Museum of Natural History, Smithsonian Institution, NHB CE 516, MRC 188, 1000 Constitution Ave NW, Washington DC 20560, USA. 4 Departamento de Zoologia, Universidade Federal do Paraná, CEP 81531-980, Curitiba, PR, 19020, Brazil. Corresponding author: Daniela Mera-Rodríguez, lmerarod@asu.edu

\begin{abstract}
The non-leaf-cutting fungus-growing ants deposited in two entomological collections in Colombia were curated and identified to assess their diversity in the country. We examined 680 specimens, identifying 41 species belonging to seven genera, bringing the total of fungus-growing ant species known from Colombia to 85. The following species are new records for Colombia: Apterostigma angustum Lattke, 1997, Mycetomoellerius jamaicensis (André, 1893), Paratrachymyrmex diversus (Mann, 1916), and Paratrachymyrmex phaleratus (Wheeler, 1925).
\end{abstract}

\section{Keywords}

Atta genus-group, Attini, biodiversity, fungus-farming ants, Neotropical ants.

Academic editor: Jason Gibbs | Received 26 July 2020 | Accepted 9 September 2020 | Published 22 September 2020

Citation: Mera-Rodríguez D, Serna F, Sosa-Calvo J, Lattke J, Rabeling C (2020) A checklist of the non-leaf-cutting fungus-growing ants (Hymenoptera, Formicidae) from Colombia, with new biogeographic records. Check List 16 (5): 1205-1227. https://doi.org/10.15560/16.5.1205

\section{Introduction}

The fungus-growing ants (tribe Attini, Atta genus-group, sensu Ward et al. 2015) are a monophyletic group endemic to the New World with the greatest diversity in the tropics, though some species may be found as far north as Long Island, New York, and as far south as Santa Cruz, Argentina (Gallardo 1915; Rabeling et al. 2007). Currently, fungus-growing ants consist of 245 valid species (Bolton 2020) in 19 genera (Li et al. 2018; Sosa-Calvo et al. 2018; Rabeling et al. 2019; Solomon et al. 2019). These ants live in an obligate mutualistic symbiosis with fungi from the Agaricaceae and Lepiotaceae families
(Hölldobler and Wilson 2011). The fungus is the main food source for most colony members (Weber 1972; Hölldobler and Wilson 1990; Mehdiabadi and Schultz 2010) and founding queens must transport mycelium from the maternal fungus garden to the newly established nest to propagate the fungus garden (Febvay and Kermarrec 1981). This fungus cultivating behavior evolved once in ants, approximately 55-65 million years ago (Nygaard et al. 2016; Branstetter et al. 2017).

Based on phylogenetic correspondence among these ants, their cultivars, and other symbionts, attine fun- 
giculture is currently divided into five distinct systems (Schultz and Brady 2008; Mehdiabadi and Schultz 2010): (i) lower agriculture, which is presumably the ancestral condition among fungus-growing ants, as practiced by the so-called Lower attines, such as Myrmicocrypta Smith, 1860 and Mycocepurus Forel, 1893; (ii) coral-fungus agriculture, practiced by species in the Apterostigma pilosum species-group, the only ones cultivating fungi outside the tribe Leucocoprineae; (iii) yeast agriculture, practiced by species in the Cyphomyrmex rimosus species-group, which cultivate nodules of yeast-like cells in a unicellular phase.; (iv) generalized higher agriculture, practiced by the non-leaf-cutting genera Mycetomoellerius Solomon et al., 2019, Paratrachymyrmex Solomon et al., 2019, Sericomyrmex Mayr, 1865, Trachymyrmex Forel, 1893, and Xerolitor Sosa-Calvo et al., 2018; and (v) leaf-cutting agriculture, considered a derived form of higher agriculture, practiced by species of the leafcutting ant genera Atta Fabricius, 1804 and Acromyrmex Mayr, 1865.

The fascinating biology of this ant-fungus-microbe symbiosis has driven researchers to study the biology and taxonomy of attine ants in Colombia for decades (Fernández and Sendoya 2004; Vergara-Navarro and Serna 2013; Fernández and Serna 2019). Parallel to this increasing research interest, the recorded number of species has also increased in recent years. Fernández and Sendoya (2004) listed at least 20 species of non-leafcutting fungus-farming ants in Colombia. VergaraNavarro and Serna (2013) recorded eleven more species of non-leaf-cutting fungus-farming ants from the central region of the country. Recently, Fernández et al. (2015) revised the 12 leaf-cutting ant species of Colombia, and in a recently published book on the ants of Colombia, Fernández and Serna (2019) recognize a total of 76 fungus-growing ant species.

This study provides a taxonomic update of the species of non-leaf-cutting fungus-growing ants known in Colombia, by examining the specimens in two entomological collections of the Universidad Nacional de Colombia: (i) the Museo Entomológico "Universidad Nacional Agronomía Bogotá" (UNAB), located at the Agricultural Sciences Department in Bogotá, and (ii) the Museo Entomológico "Francisco Luis Gallego" (MEFLG) located at the Sciences Department in Medellín. The UNAB collection was established in 2001 and contains more than 200,000 specimens representing 20 orders of Hexapoda (Martinez-Alava and Serna 2015; Serna et al. 2017). The Museo Entomológico "Francisco Luis Gallego" was established in 1937 and houses around 350,000 insect specimens (Serna et al. 2017). We examined a total of 680 specimens, recognizing 41 non-leaf-cutting fungus-growing ant species in Colombia, including four new records and five potentially new species.

\section{Methods}

Ant specimens were curated following the protocol in Martínez-Alava and Serna (2015). Alcohol-preserved specimens were individually point-mounted, labelled with the corresponding collecting data, and placed into Ward boxes. Each box was then tagged with the species name, and a unique identifier catalog number per taxon.

Morphological terminology follows Serna and Mackay (2010), Serna et al. (2011), Lattke (1997), and Delsinne et al. (2019). Specimens were identified to genus level employing the taxonomic studies of Serna and Vergara-Navarro (2001), Fernández and Serna (2019) and Solomon et al. (2019). Species identification was achieved using published and unpublished taxonomic keys for Apterostigma Mayr, 1865 (Lattke 1997); Cyphomyrmex Mayr, 1862 (Kempf 1967; Snelling and Longino 1992; Sanhudo 2011; Albuquerque 2014); Mycocepurus (Kempf 1963; Mackay et al. 2004); Myrmicocrypta (Sosa-Calvo 2015); Sericomyrmex (Ješovnik and Schultz 2017); Paratrachymyrmex and Mycetomoellerius (Mayhé-Nunes and Brandão 2002, 2005, 2007; Rabeling et al. 2007; Solomon et al. 2019). Specimens were studied with a Leica M205 C stereomicroscope and were compared with specimens deposited in the Social Insects Biodiversity Repository at Arizona State University in Tempe, AZ, U.S.A. (ASU-SIBR) and to images available on Antweb database (https://www.antweb.org). Most of the images used to illustrate species in this study (Figs 2-10) were obtained from Antweb with permission, and the photographer and specimen codes are specified in each figure caption. All other composite images were generated using a Leica DFC450 digital camera, mounted onto a Leica M205 C stereomicroscope and stacked with either the Leica Application Suite (Version 4.5) or the Helicon Focus (Version 6.6.1) software packages. Species distribution maps were created with QGIS Version 3.14.0 software (QGIS 2020).

\section{Results}

We studied 680 fungus-growing ant specimens representing 41 species in seven genera: Apterostigma (18 species), Cyphomyrmex (8 spp.), Mycetomoellerius (3 spp.), Mycocepurus (1 sp.), Myrmicocrypta (2 spp.), Paratrachymyrmex (5 spp.), and Sericomyrmex (4 spp.). Four species are new records for Colombia: Apterostigma angustum Lattke, 1997, Mycetomoellerius jamaicensis (André, 1893), Paratrachymyrmex diversus (Mann, 1916), and Paratrachymyrmex phaleratus (Wheeler, 1925).

We also identified five species that are potentially new to science, three in Apterostigma, one in Myrmicocrypta, and one in Mycetomoellerius. These species will eventually be described in ongoing revisionary studies of the respective genera.

Departments surveyed. We recorded non-leaf-cutting fungus-farming ants in 38 municipalities from 17 departments (Fig. 1A-D): Amazonas, Antioquia, Atlántico, 

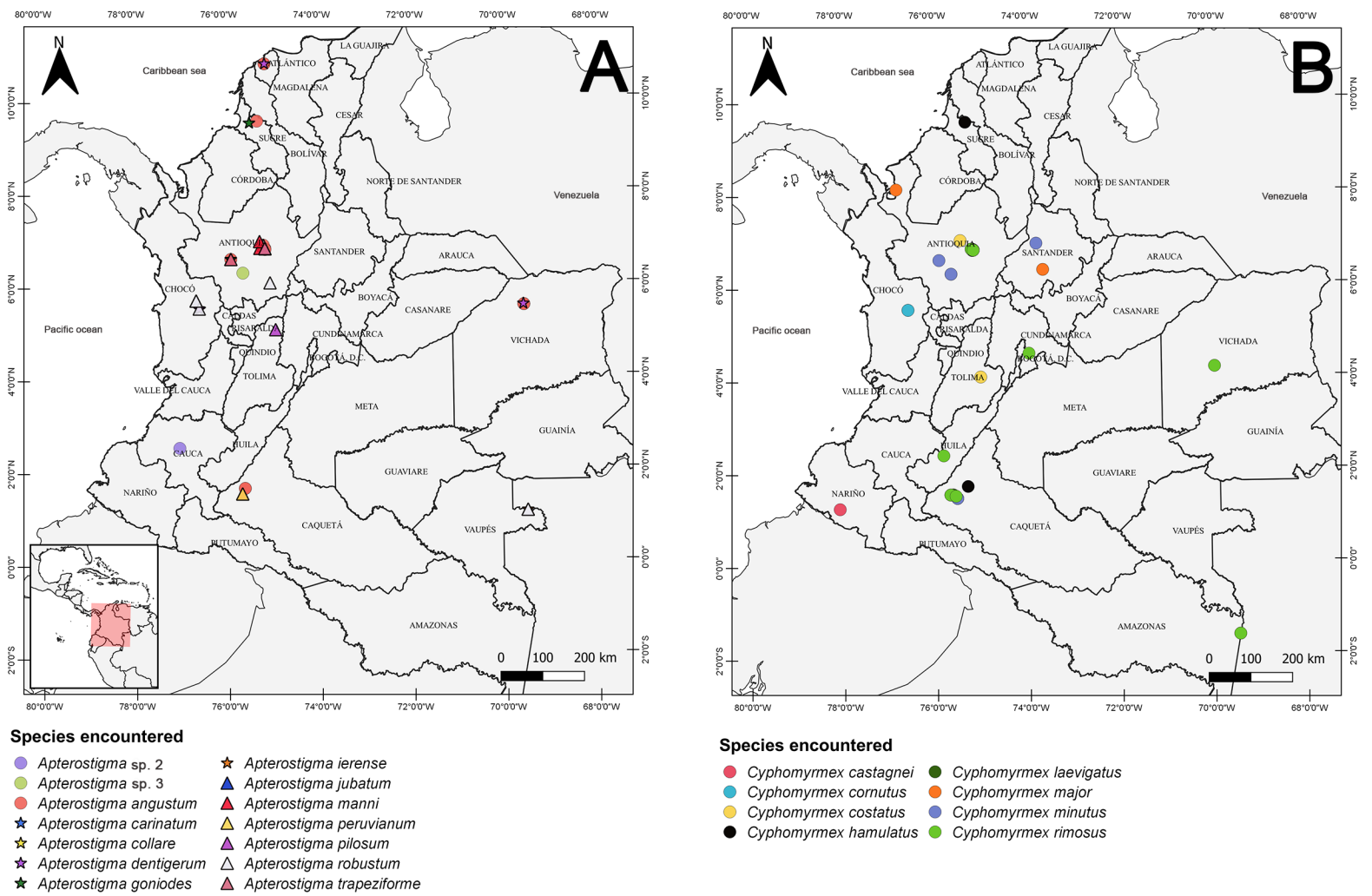

Apterostigma trapeziforme
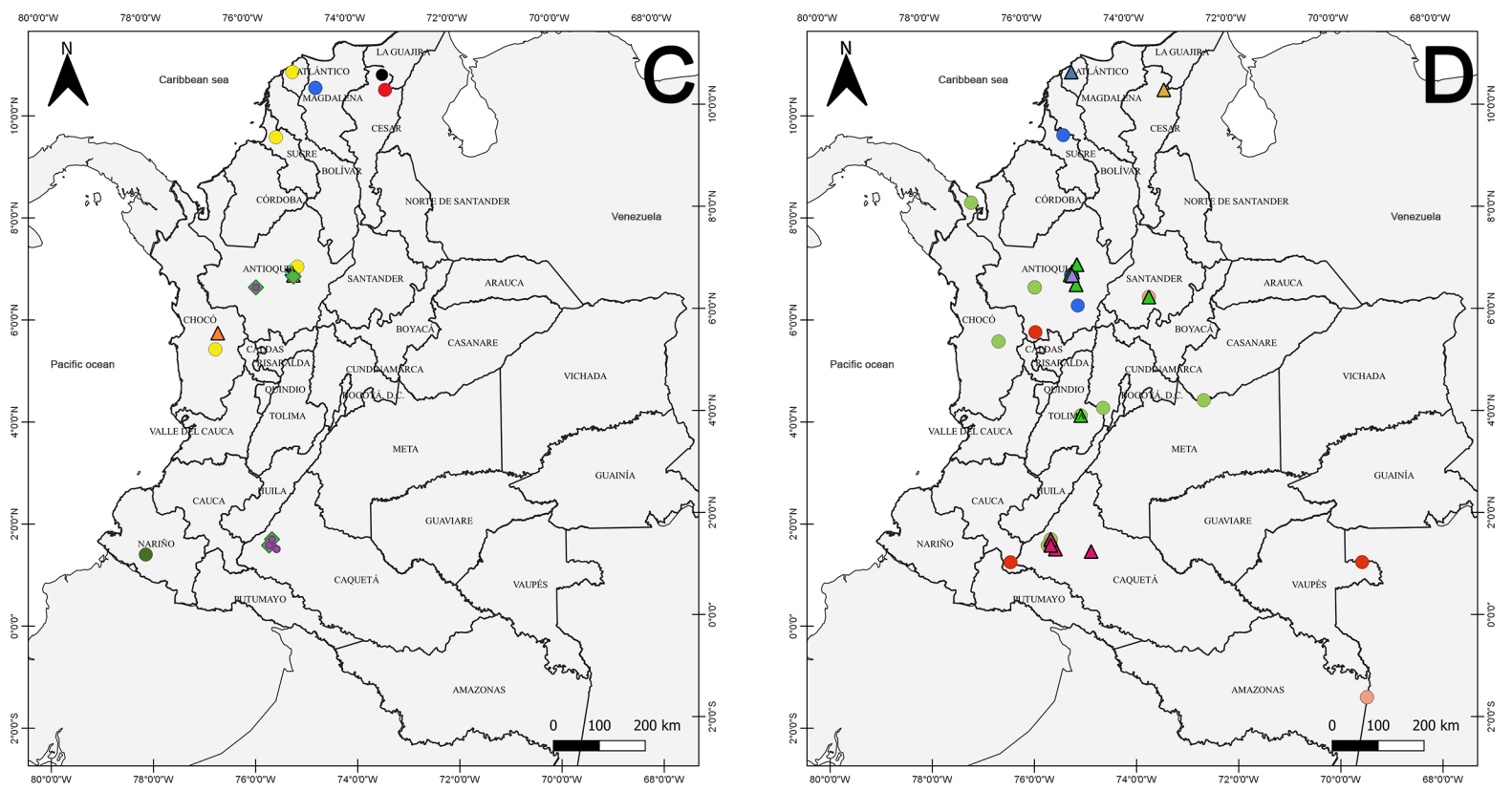

Species encountered

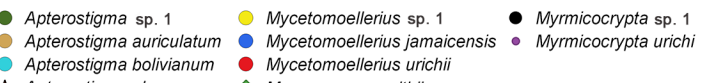

Apterostigma bolivianum Mycetomoellerius urichii

* Apterostigma chocoense $\diamond$ Mycocepurus smithii

Apterostigma chocoense

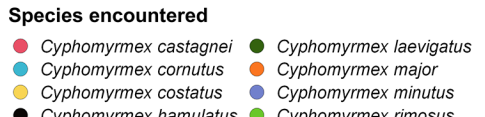

Cyphomyrmex costatus Cyphomyrmex minutus

Species encountered

- Sericomyrmex amabilis

- Sericomyrmex bondar

Sericomyrmex mayri

Pericomyrmex saussurei $\triangle$ Paratrachymyrmex cornetzi
$\Delta$ Paratrachymyrmex diversus
$\triangle$ Paratrachymyrmex irmgardae
$\triangle$ Paratrachymyrmex phaleratus

Figure 1. Geographic distribution of non-leaf-cutting fungus-growing ant species in Colombia. A. Apterostigma pilosum species-group. B. Genus Cyphomyrmex. C. Apterostigma auriculatum species-group, and genera Mycocepurus, Myrmicocrypta, and Mycetomoellerius. D. Genera Sericomyrmex and Paratrachymyrmex.

Caquetá, Cauca, Cesar, Chocó, Cundinamarca, Guaviare, Huila, Meta, Nariño, Putumayo, Santander, Sucre, Tolima, and Vaupés. Sericomyrmex amabilis Wheeler, 1925 is the most widely distributed species in the country, being present in six departments.
Elevational distribution. Specimens were collected in localities ranging from sea level up to $1927 \mathrm{~m}$, with an average of $572 \mathrm{~m}$, though most of the species (90\%) were found in locations below $650 \mathrm{~m}$. However, some species such as Cyphomyrmex major Forel, 1901, Cypho- 
myrmex rimosus (Spinola, 1851), and Sericomyrmex bondari Borgmeier, 1937 show an extended altitudinal distribution: 1-1426 m, $280-1927 \mathrm{~m}$, and 85-1700 m, respectively.

\section{Examined material.}

Apterostigma Mayr, 1865

\section{(1) Apterostigma angustum Lattke, 1997 Figure 2A, B}

Material examined. COLOMBIA: Antioquia • 4 workers; Amalfi; 06 $46^{\prime} 66^{\prime \prime} \mathrm{N}, 075^{\circ} 06^{\prime} 42^{\prime \prime} \mathrm{W}$; alt. $1045 \mathrm{~m}$; 25 Jun. 1997; F. Serna leg.; UNAB 4365; Antioquia • 6 workers; Amalfi; $06^{\circ} 51^{\prime} 26^{\prime \prime} \mathrm{N}, 075^{\circ} 07^{\prime} 32^{\prime \prime} \mathrm{W}$; alt. 1000 m; 30 Jul. 1997; F. Serna leg.; MEFLG 5786; Antioquia - 2 workers; Amalfi; $06^{\circ} 45^{\prime} 42.43^{\prime \prime} \mathrm{N}, 075^{\circ} 05^{\prime} 28.31^{\prime \prime} \mathrm{W}$; alt. 1221 m; Sep. 2006; M. A. Vanegas leg.; MEFLG 11086; Antioquia • 2 workers; Santafé de Antioquia; $06^{\circ}$ $33^{\prime} 41^{\prime \prime} \mathrm{N}, 075^{\circ} 49^{\prime} 54^{\prime \prime} \mathrm{W}$; alt. 600 m; 06 Oct. 2000; E. Vergara, F. Serna leg.; UNAB 4738; Antioquia - 12 gynes, 20 workers; Amalfi; 06 $51^{\circ} 26^{\prime \prime} \mathrm{N}, 075^{\circ} 07^{\prime} 32^{\prime \prime} \mathrm{W}$; alt. 1000 m; 30 Jul. 1997; F. Serna leg.; MEFLG 5786; Atlántico - 1 worker; Juan de Acosta; $10^{\circ} 46^{\prime} 02^{\prime \prime} \mathrm{N}, 075^{\circ}$ 02'34"W; alt. 117-250 m; Feb.-May 2012; J. Castro, S. Royero leg.; UNAB 5457; Caquetá • 8 workers; Florencia; $01^{\circ} 37^{\prime} 11^{\prime \prime} \mathrm{N}, 075^{\circ} 36^{\prime} 14^{\prime \prime} \mathrm{W}$; alt. 300 m; 31 Mar. 2016; F. Serna leg.; UNAB 4365; Chocó • 1 worker; Lloró; $05^{\circ}$ $29^{\prime} 00^{\prime \prime} \mathrm{N}, 076^{\circ} 32^{\prime} 00^{\prime \prime} \mathrm{W}$; alt. 115 m; 04 Mar. 2001; J. Neita leg.; UNAB 4366; Chocó • 4 workers; Lloró; 05²9'00"N, $076^{\circ} 32^{\prime} 00^{\prime \prime} \mathrm{W}$; alt. $115 \mathrm{~m}$; 03 Nov. 2000; J. Neita leg.; UNAB 4366; Sucre • 1 worker; Ovejas; 09³2'4.1"N, $075^{\circ}$ 13'18.5"W; alt. 277 m; 4 Feb. 2016; H. Cadena leg.; MEFLG 40895.

Identification. Frontal lobes in frontal view triangular; general cephalic profile in lateral view ovoid; compound eyes relatively small; and ventral mesopleural carina (epicnemial carina of Serna and Mackay 2010) absent (Lattke 1997).

Distribution. The specimens found in Colombia are all new records for the country as well as a major range expansion to the west (Fig. 1A) as Apterostigma angustum was only known from Venezuela and French Guiana (Lattke 1997; Fernández and Sendoya 2004).

\section{(2) Apterostigma auriculatum Wheeler, 1925} Figure 2C, D

Material examined. COLOMBIA: Antioquia - 1 worker; Amalfi; 06 $54^{\prime} 11^{\prime \prime} \mathrm{N}, 075^{\circ} 05^{\prime} 26.7^{\prime \prime} \mathrm{W}$; alt. $1045 \mathrm{~m}$; 20 Apr. 1998; F. Serna leg.; UNAB 4361.

Identification. Eyes relatively big and prominent (more than nine ommatidia along the shortest axis); frontal lobes subquadrate in full-face view, anterior margin of clypeus concave, lacking shiny cuticular strip; neck relatively long and broad, lacking median carina; humeral prominence in dorsal view shaped as angular lobe; ventral mesopleural carina in lateral view fine; and petiole in lateral view triangular, with antero- and posterodorsal profiles almost of same length (Lattke 1997).

Distribution. This species occurs from Honduras to Mato Grosso do Sul in Brazil and is widely distributed throughout central and northwestern South America (Lattke 1997; Fernández and Sendoya 2004).

\section{(3) Apterostigma bolivianum Weber, 1938 \\ Figure 2E, F}

Material examined. COLOMBIA: Antioquia 1 worker; Amalfi; 06 $51^{\prime} 26^{\prime \prime} \mathrm{N}, 075^{\circ} 07^{\prime} 32^{\prime \prime} \mathrm{W}$; alt. $1000 \mathrm{~m} ; 30$ Jul. 1997; F. Serna leg.; MEFLG 40854.

Identification. Eyes prominent (more than nine ommatidia along the shortest axis); frontal lobes in full-face view subquadrate; anterior margin of clypeus in fullface view concave, lacking shiny cuticular strip; neck lacking median carina; mandibles elongate; anterior margin of pronotum in dorsal view convex, with median emargination and no lateral thickening; and mesopleural ventral carina in lateral view well-developed, thick (Lattke 1997).

Distribution. In Colombia, A. bolivianum was only known from Amazonas, thus the new record from Antioquia (Fig. 1C) suggests this species might be broadly distributed throughout the country (Fig. 1C) (Weber 1938; Kempf 1972; Fernández et al. 1996; Lattke 1997). Apterostigma bolivianum is also known from the following countries in northwestern South America: Bolivia, Ecuador, Peru, and Venezuela.

\section{(4) Apterostigma carinatum Lattke, 1997}

Figure 2G, H

Material examined. COLOMBIA: Chocó - 1 worker; Lloró; 05³0'39"N, 76³2'40"W; alt. 115 m; 12 Dec. 2001; Y. Palacios, O. Mena leg.; UNAB 5458; Chocó • 1 worker; Lloró; $05^{\circ} 30^{\prime} 00^{\prime \prime} \mathrm{N}, 076^{\circ} 31^{\prime} 00^{\prime \prime} \mathrm{W}$; alt. $97 \mathrm{~m} ; 18$ Nov. 2002; O. Mena, Y. Palacios leg.; UNAB 5458; Vaupés - 1 worker; Taraira; $01^{\circ} 04^{\prime} 00^{\prime \prime} \mathrm{S}, 069^{\circ} 31^{\prime} 00^{\prime \prime} \mathrm{W}$; alt. 85 m; 4-20 May 2001; A. Sabogal leg.; UNAB 5458.

Identification. Frontal lobe in full-face view either convex or triangular, with rounded apex; anterior margin of clypeus in full-face view convex, with shiny cuticular strip; dorsal mesosomal carina present, in lateral view low and uniform; petiole in lateral view elongate; dorsal and ventral curvatures of gaster in lateral view similar in size; lateral carina of gaster inconspicuous; and metacoxa with dorsoposterior keel (Lattke 1997).

Distribution. This species is known to occur in Ecuador and the Pacific coast of Colombia. The present record from Vaupés expands its geographic distribution to the Amazonian region of the country (Fig. 1A) (Lattke 1997; Solar et al. 2016).

\section{(5) Apterostigma chocoense Lattke, 1997}

Figure 3A, B

Material examined. COLOMBIA: Antioquia • 9 work- 


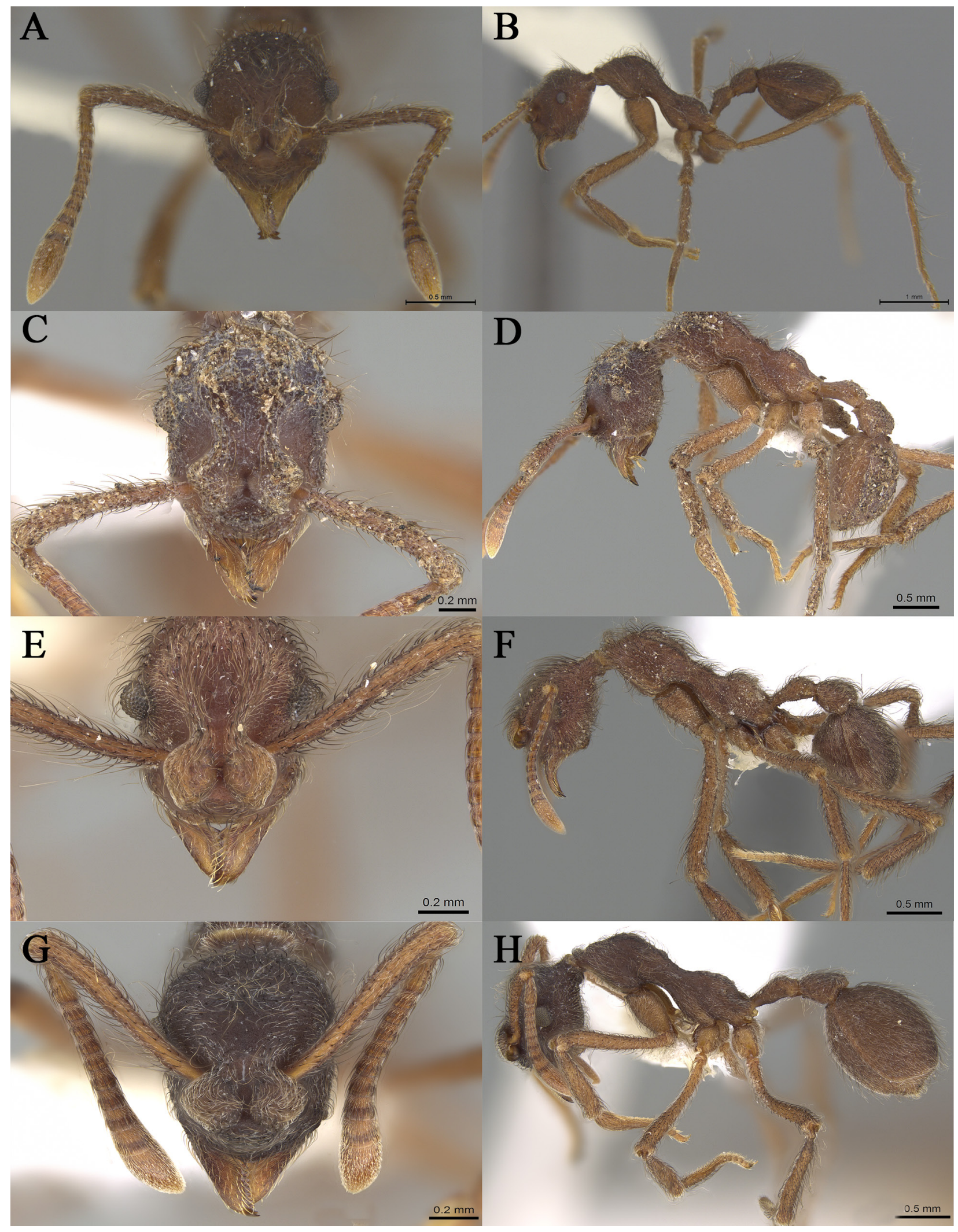

Figure 2. Apterostigma species encountered in Colombia. A, B. Apterostigma angustum, J. Sosa-Calvo. C-H. Figures taken by M. Esposito obtained from the Antweb database (http://www.antweb.org). C, D. Apterostigma auriculatum, CASENT0922035. E, F. Apterostigma bolivianum, CASENT0922036. G, H. Apterostigma carinatum, CASENT0922038.

ers; Amalfi; $06^{\circ} 51^{\prime} 26^{\prime \prime} \mathrm{N}, 075^{\circ} 07^{\prime} 32^{\prime \prime} \mathrm{W}$; alt. $1000 \mathrm{~m} ; 30$ Jul. 1997; F. Serna leg.; MEFLG 40855, 40856, 40857.

Identification. Triangular propodeal denticles present; ventral profile of gaster convex anteriorly and concave posteriorly; and ventral profile of gaster in lateral view sinuous (Lattke 1997).

Distribution. The present record expands the species known distribution in Colombia from the northwestern 


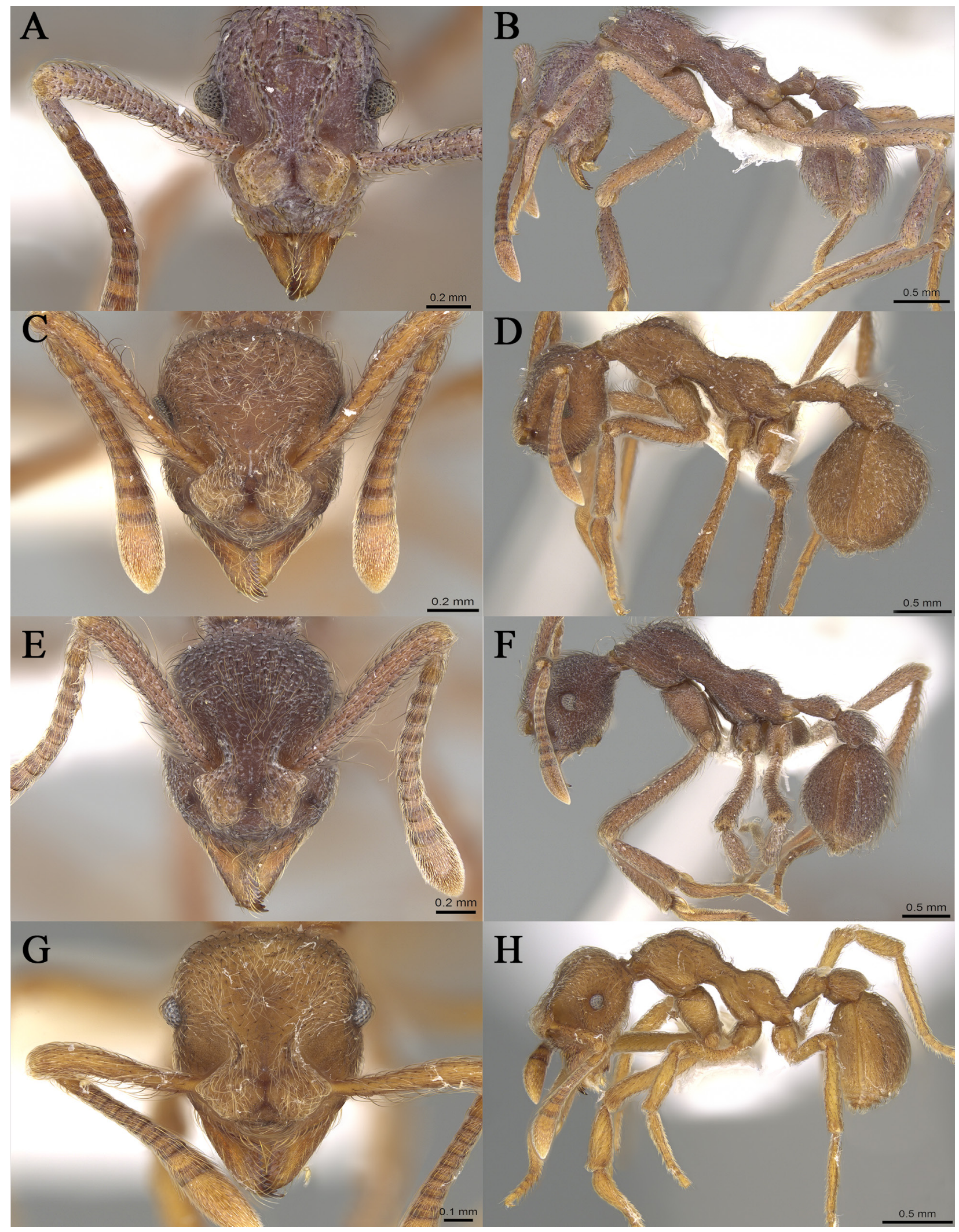

Figure 3. Apterostigma species encountered in Colombia. A, B. Apterostigma chocoense, CASENT0922037. C, D. Apterostigma collare, CASENT0922041. E, F. Apterostigma dentigerum, CASENT0922039. G, H. Apterostigma goniodes, CASENT0922040. Photographs taken by M. Esposito obtained from the Antweb database (http://www.antweb.org).

Pacific coast to the Andean region (Fig. 1C). Outside of Colombia this species is known in Costa Rica, French Guiana, and Panama (Brandão 1991; Lattke 1997; Fernández and Sendoya 2004).
(6) Apterostigma collare Emery, 1896

Figure 3C, D

Material examined. COLOMBIA: Chocó $・ 1$ worker; Lloró; $05^{\circ} 29^{\prime} 00^{\prime \prime} \mathrm{N}, 076^{\circ} 32^{\prime} 00^{\prime \prime} \mathrm{W}$; alt. $115 \mathrm{~m}$; 15 Mar. 
2002; Y. Palacios, O. Mena leg.; UNAB 4739.

Identification. Frontal lobes in frontal view subquadrate, with lateroventral margin curved posteriorly; anterior clypeal margin with a median angle; gaster in lateral view subspherical, with inconspicuous lateral carina; and body lacking erect hairs (Lattke 1997).

Distribution. Previous Colombian records are from the departments of Cauca, Valle del Cauca, and Magdalena. This new record from Chocó indicates the species might be widely distributed across the Pacific and northern coasts of the country (Fig. 1A). Apterostigma collare is known from several countries in Central America: Costa Rica, Honduras, Mexico (Veracruz), Nicaragua, and Panama. In South America it has been found only in Colombia and Venezuela (Lattke 1997).

\section{(7) Apterostigma dentigerum Wheeler, 1925}

Figure 3E, F

Material examined. COLOMBIA: Atlántico • 1 worker; Juan de Acosta; $10^{\circ} 46^{\prime} 2^{\prime \prime} \mathrm{N}, 075^{\circ} 02^{\prime} 34^{\prime \prime} \mathrm{W}$; alt. $177-250$ m; Feb.-May 2012; J. Castro, S. Royero leg.; UNAB 4362; Chocó - 2 workers; Lloró; $05^{\circ} 29^{\prime} 00^{\prime \prime} \mathrm{N}, 076^{\circ}$ $32^{\prime} 00^{\prime \prime} \mathrm{W}$; alt. 115 m; 12 Dec. 2001; Y. Palacios, O. Mena leg.; UNAB 4740; Chocó • 1 worker; Lloró; $05^{\circ} 29^{\prime} 00^{\prime \prime} \mathrm{N}$, $076^{\circ} 32^{\prime} 00^{\prime \prime} \mathrm{W}$; alt. $115 \mathrm{~m}$; 04 May 2002; Y. Palacios, O. Mena leg.; UNAB 2230.

Identification. Frontal lobes in frontal view with strong angle or posterolateral lobe; lateral-ventral margin of frontal lobe sinusoidal: convex posteriorly and curved towards dorsum, without any ventral curvature; and propodeum in lateral view with anterodorsal profile long and gradually very convex, posteriorly shorter and more curved (Lattke 1997).

Distribution. Apterostigma dentigerum is widely distributed in Central and South America, its known distribution range extends from Honduras to Mato Grosso in central Brazil (Lattke 1997; Mayhé-Nuñes and Jaffé 1998).

\section{(8) Apterostigma goniodes Lattke, 1997}

Figure $3 \mathrm{G}, \mathrm{H}$

Material examined. COLOMBIA: Antioquia $\cdot 5$ workers; Santafé de Antioquia; 06 $33^{\prime} 41^{\prime \prime} \mathrm{N}, 075^{\circ} 49^{\prime} 54^{\prime \prime} \mathrm{W}$; alt. 600 m; 06 Oct. 2000; E. Vergara, F. Serna leg.; UNAB 4772; Antioquia - 2 workers; Santafé de Antioquia; $06^{\circ}$ $33^{\prime} 41^{\prime \prime} \mathrm{N}, 075^{\circ} 49^{\prime} 54^{\prime \prime} \mathrm{W}$; alt. $600 \mathrm{~m}$; 06 Oct. 2000; E. Vergara, F. Serna leg.; UNAB 4772; Sucre • 1 worker; Colosó; 09²9'44"N, 075²3'17"W; alt. 200-700 m; 30 Aug. 2009; G. Mercado leg.; UNAB 4741; Sucre • 2 workers; Colosó; $09^{\circ} 29^{\prime} 44^{\prime \prime} \mathrm{N}, 075^{\circ} 23^{\prime} 17^{\prime \prime} \mathrm{W}$; alt. 200-700 m; 29 Aug. 2009; G. Mercado leg.; UNAB 4741.

Identification. Frontal lobes in frontal view triangular, each posterior margin forms an (almost) right angle with the lateral margin; ventral mesopleural carina well-developed and lamelliform; and longitudinal translucent keel present on the postpetiolar sternite (Lattke 1997).

Distribution. Apterostigma goniodes is distributed from southern Mexico throughout Central America to Colombia (Lattke 1997; Castro et al. 2018; García-Cárdenas et al. 2018).

\section{(9) Apterostigma ierense Weber, 1937 \\ Figure 4A, B}

Material examined. COLOMBIA: Vaupés 1 worker; Taraira; $01^{\circ} 04^{\prime} 00^{\prime \prime} \mathrm{S}, 069^{\circ} 31^{\prime} 00^{\prime \prime} \mathrm{W}$; alt. $85 \mathrm{~m}$; $14-20$ May 2001; A. Sabogal leg.; UNAB 4742.

Identification. Lateroventral margin of frontal lobes completely curved and covering the antennae insertions; the cervical carina in dorsal view is shaped as two rounded lobes; and body densely pubescent (Lattke 1997).

Distribution. Apterostigma ierense is mostly distributed in the Amazon basin, being reported from Bolivia, Brazil, Colombia, Ecuador, Peru, and Venezuela. In Central America, A. ierense is only known from Panama (Kempf 1972; Lattke 1997; Mayhé-Nuñes and Jaffé 1998; Fernández and Sendoya 2004).

\section{(10) Apterostigma jubatum Wheeler, 1925}

Figure 4C, D

Material examined. COLOMBIA: Chocó 1 worker; Quibdó; $05^{\circ} 40^{\prime} 40^{\prime \prime} \mathrm{N}, 076^{\circ} 35^{\prime} 36^{\prime \prime} \mathrm{W}$; alt. $35 \mathrm{~m}$; 24 Nov. 2001; J. Neita leg.; UNAB 4743.

Identification. Head in frontal view with frontal lobes ovoid; anterior portion of clypeus with profile concave and convex in the middle; mandibles with 7-8 teeth; and humeral prominence high (Lattke 1997).

Distribution. Apterostigma jubatum is mostly distributed throughout the Amazon-Orinoco basin, known to occur in Bolivia, Colombia, Peru, and Venezuela (Fernández et al. 1996; Lattke 1997) as well as the Brazilian states of Amazonas, Minas Gerais, and Pará.

\section{(11) Apterostigma manni Weber, 1938}

Figure 4E, F

Material examined. COLOMBIA: Antioquia - 2 workers; Amalfi; $06^{\circ} 55^{\prime} 00^{\prime \prime} \mathrm{N}, 075^{\circ} 04^{\prime} 00^{\prime \prime} \mathrm{W}$; alt. $990 \mathrm{~m}$; 30 Jul. 1997; F. Serna leg.; UNAB 4745; MEFLG 5787; Antioquia 1 worker; Santafé de Antioquia; $06^{\circ} 33^{\prime} 41^{\prime \prime} \mathrm{N}$, $075^{\circ} 49^{\prime} 54^{\prime \prime} \mathrm{W}$; alt. $600 \mathrm{~m}$; 06 Oct. 2000; E. Vergara, F. Serna leg.; UNAB 4744; Caquetá • 1 worker; Florencia; $01^{\circ} 30^{\prime} 4^{\prime \prime} \mathrm{N}, 075^{\circ} 39^{\prime} 42^{\prime \prime} \mathrm{W}$; alt. $280 \mathrm{~m}$; 01 Sep. 2017; UNAB members leg.; UNAB 4745.

Identification. Eyes reniform; mesopleural carina welldeveloped and lamelliform; postpetiole usually with ventral lamelliform longitudinal carina; body yellowish or light brown; and abdominal sternite IV with anteromedian transverse carina (Lattke 1997).

Distribution. Apterostigma manni is present in southern Central America (Costa Rica and Panama), northwestern South America (Bolivia, Colombia, Peru, and Venezuela), and in the Brazilian states of Bahia and Minas Gerais (Lattke 1997; Fernández and Sendoya 2004). 


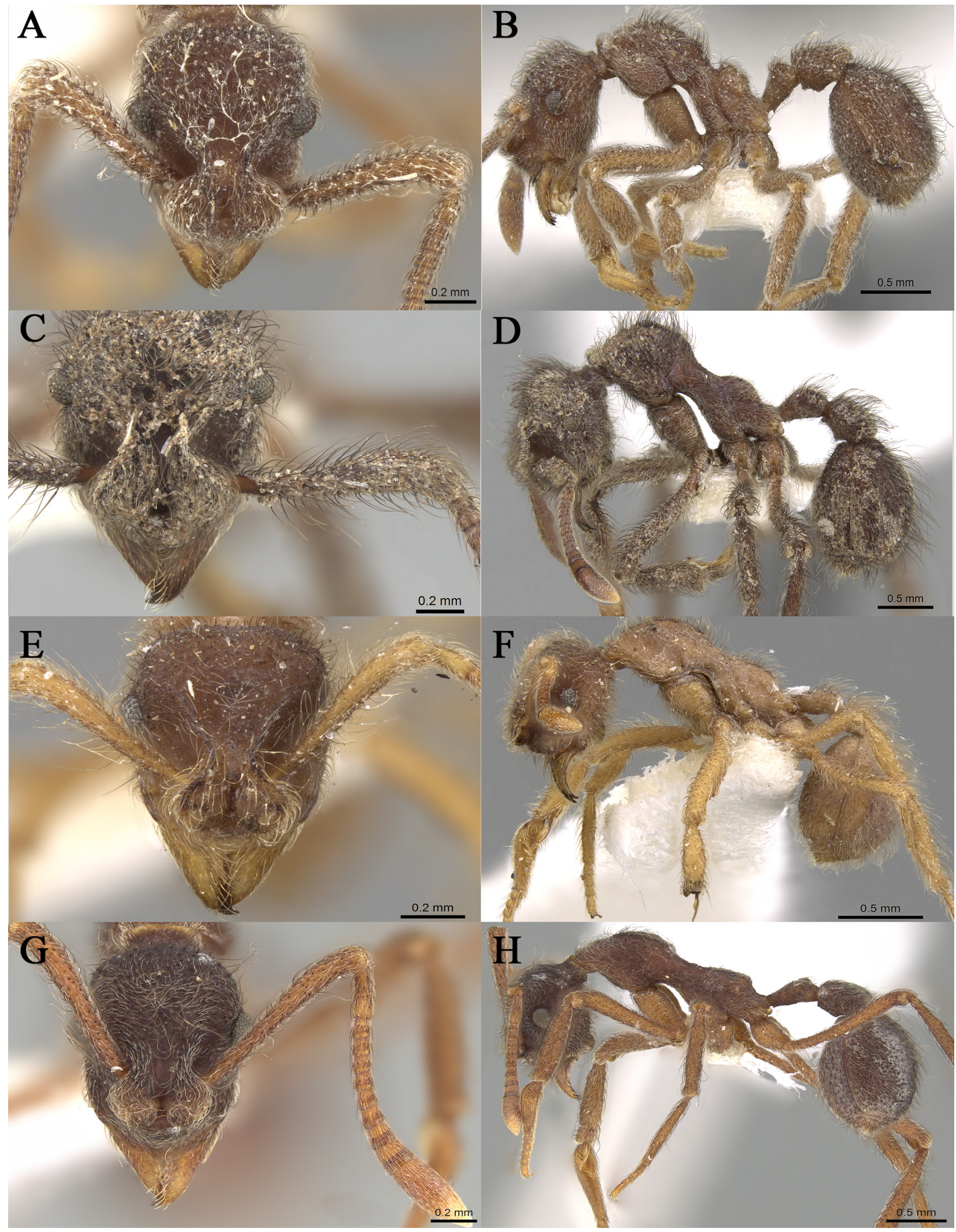

Figure 4. Apterostigma species encountered in Colombia. A, B. Apterostigma ierense, CASENT0281772. C, D. Apterostigma jubatum, CASENT0281773. E, F. Apterostigma manni, CASENT0281776. G, H. Apterostigma peruvianum, CASENT0922046. Photographs taken by S. Hartman (A-F) and M. Esposito (G-H) obtained from the Antweb database (http://www.antweb.org).

(12) Apterostigma peruvianum Wheeler, 1925

Figure $4 \mathrm{G}, \mathrm{H}$

Material examined. COLOMBIA: Caquetá • 3 workers; Florencia; $01^{\circ} 30^{\prime} 4^{\prime \prime} \mathrm{N}, 075^{\circ} 39^{\prime} 42^{\prime \prime} \mathrm{W}$; alt. 280 m; Sep.
2017; UNAB members leg.; UNAB 4737.

Identification. Frontal lobes in frontal view subquadrate, each posterior margin straight and inclined, anterolateral margins convex; posterior cephalic profile rounded; eyes 
with a maximum of 10 ommatidia along the shortest axis; and postpetiole with posteroventral process relatively big and usually dentiform (Lattke 1997).

Distribution. Previous Colombian records are from Valle del Cauca, thus this new record from Caquetá expands the distribution of this species from the Andes into the Amazon region (Fig. 1A). Besides Colombia, Apterostigma peruvianum is also present in Bolivia, Ecuador, Peru, and Venezuela (Kempf 1972; Lattke 1997; Fernández and Sendoya 2004).

\section{(13) Apterostigma reburrum Lattke, 1997}

Figure 5A, B

Material examined. COLOMBIA: Antioquia - 1 worker; Amalfi; 06 $55^{\prime} 00^{\prime \prime} \mathrm{N}, 075^{\circ} 04^{\prime} 00^{\prime \prime} \mathrm{W}$; alt. $1045 \mathrm{~m}$; 20 Apr. 1998; F. Serna leg.; UNAB 5454; Chocó • 1 worker; $05^{\circ} 40^{\prime} 40^{\prime \prime} \mathrm{N}, 076^{\circ} 35^{\prime} 36^{\prime \prime} \mathrm{W}$; alt. $35 \mathrm{~m} ; 24$ Nov. 2001; J. Neita leg.; UNAB 5454.

Identification. Subocular prominence higher than eye in frontal view of head; anteroventral petiolar denticle welldeveloped; body with abundant semi-erect thick hairs; propodeum without denticles; and cephalic neck short and lacking carina (Lattke 1997).

Distribution. Besides the Colombian departments of Antioquia, Valle del Cauca, and San Andres Island, A. reburrum is only known from French Guiana (Lattke 1997; Fichaux et al. 2019), but this disjunct distribution suggests the species might be more widely distributed across northern South America and possibly the Caribbean.

\section{(14) Apterostigma robustum Emery, 1896}

Figure 5C, D

Material examined COLOMBIA: Antioquia • 1 worker; San Luis; 0602'33.7"N, 07459'39.9"W; alt. 1005 m; Mar. 1992; F. Serna leg.; MEFLG 5170; Chocó • 4 workers; Quibdó; 05 $40^{\prime} 40^{\prime \prime} \mathrm{N}, 076^{\circ} 35^{\prime} 36^{\prime \prime} \mathrm{W}$; alt. $35 \mathrm{~m} ; 23$ Nov. 2001; J. Neita leg.; UNAB 4747; Vaupés • 3 gynes, 6 workers; Taraira; $01^{\circ} 04^{\prime} 00^{\prime \prime} \mathrm{S}, 069^{\circ} 31^{\prime} 00^{\prime \prime} \mathrm{W}$; alt. $85 \mathrm{~m}$; 14-20 May 2001, A. Sabogal leg.; UNAB 4746.

Identification. Frontal lobes with each lateral margin convex and strongly curved ventrally; anterior margin of clypeus uniformly convex; mandible with 11 teeth that increase gradually in size towards the apical tooth; and propodeum in lateral view gradually higher than the metanotum (Lattke 1997).

Distribution. Apterostigma robustum is distributed from Honduras in Central America south to Bolivia and Brazil, where it occurs in the states of Amazonas, Maranhão, and Pará (Kempf 1972; Lattke 1997; Fernández and Sendoya 2004).

\section{(15) Apterostigma pilosum species complex}

Figure 5E, F

Material examined. COLOMBIA: Antioquia • 5 workers; Amalfi; $06^{\circ} 55^{\prime} 00^{\prime \prime} \mathrm{N}, 075^{\circ} 04^{\prime} 00^{\prime \prime} \mathrm{W}$; alt. $1045 \mathrm{~m} ; 25$ Jun. 1997; F. Serna leg.; UNAB 5453; Antioquia • 2 workers; Amalfi; $06^{\circ} 46^{\prime} 39^{\prime \prime} \mathrm{N}, 075^{\circ} 05^{\prime} 22^{\prime \prime} \mathrm{W}$; alt. $1550 \mathrm{~m}$;
30 Jul. 1997; F. Serna leg.; MEFLG 40843; Antioquia • 1 worker; Santafé de Antioquia; 06 33'41"N, 075 49'45"W; alt. 600 m; 06 Oct. 2000; E. Vergara, F. Serna leg.; UNAB 5453; Tolima • 1 worker; Armero; 0501'55"N, 07453'27"W; alt. 261 m; 12 Apr. 2012; G. Villamizar leg.; UNAB 5453.

Identification. The Apterostigma pilosum species-complex needs a thorough taxonomic revision. Lattke (1997) provides a general diagnosis for the putative species in the complex: eyes with 5-6 ommatidia along the minor axis; mesopleural ventral carina present or absent but never prominent, lamelliform, nor thick and shining; frontal lobes in cephalic dorsal view triangular or uniformly convex; anterolateral mesonotal prominence absent; and dorsal postpetiolar profile in lateral view strongly curved. The above specimens all agree with this diagnosis.

Distribution. The species of the Apterostigma pilosum complex are widely distributed in Central and South America, ranging from Tamaulipas in Mexico south to Buenos Aires, Argentina.

\section{(16) Apterostigma sp. 1, auriculatum species-group}

Material examined. COLOMBIA: Nariño • 1 worker; Barbacoas; $01^{\circ} 21^{\prime} 49^{\prime \prime} \mathrm{N}, 078^{\circ} 04^{\prime} 45^{\prime \prime} \mathrm{W}$; alt. $640 \mathrm{~m}$; 25-29 Jul. 2006; A. Miranda leg.; UNAB 5459.

Comment. This presumably new species is only known from a single worker and will be described in a forthcoming taxonomic revision of the $A$. auriculatum species-group.

\section{(17) Apterostigma sp. 2, pilosum species-group}

Material examined. COLOMBIA: Cauca - 1 worker; Tambo; 02 $30^{\prime} 24.11^{\prime \prime} \mathrm{N}, 076^{\circ} 59^{\prime} 57^{\prime \prime} \mathrm{W}$; alt. $1448 \mathrm{~m}$; 1-8 Apr. 2006; A. Galvis, Y. Mena leg.; UNAB 5455.

Comment. This new Apterostigma species is only known from a single worker and will be described in a forthcoming taxonomic study.

\section{(18) Apterostigma sp. 3, pilosum species-group}

Material examined. COLOMBIA: Antioquia • 1 worker; Medellín; 06² $15^{\prime} 44.4^{\prime \prime} \mathrm{N}, 075^{\circ} 34^{\prime} 33.4^{\prime \prime} \mathrm{W}$; alt. 1460 m; May 1999; MEFLG members leg.; MEFLG 5786.

Comment. This new Apterostigma species is only known from a single worker and will be described in a forthcoming taxonomic treatment.

Cyphomyrmex Mayr, 1862

(19) Cyphomyrmex castagnei Mackay \& Baena, 1993

Material examined. COLOMBIA: Nariño • 1 worker; Ricaurte; $01^{\circ} 13^{\prime} 35^{\prime \prime} \mathrm{N}, 078^{\circ} 03^{\prime} 04^{\prime \prime} \mathrm{W}$; alt. $920 \mathrm{~m}$; 21 Sep. 2015; J. Cancino leg.; UNAB 4748.

Identification. Mandible with five teeth; lateral corner of cephalic vertex with long horn-like extensions; 


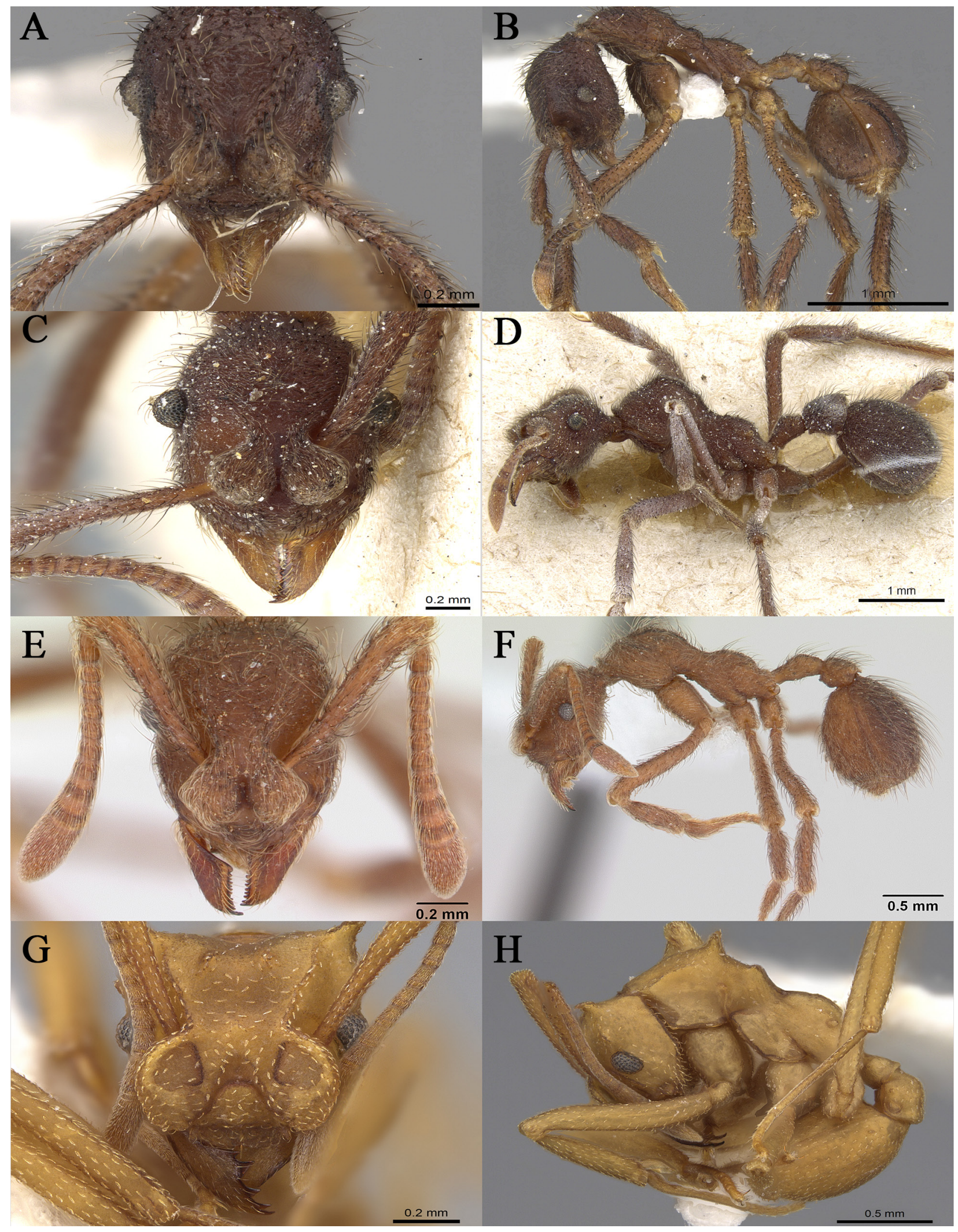

Figure 5. Apterostigma and Cyphomyrmex species encountered in Colombia. A, B. Apterostigma reburrum, CASENT0901686/ R. Perry. C, D. Apterostigma robustum, CASENT0904977/ Z. Lieberman. E, F. Apterostigma pilosum complex, CASENT0603533/ A. Nobile. G, H. Cyphomyrmex cornutus, CASENT0923103/ W. Lee. Photographs obtained from the Antweb database (http://www.antweb.org).

mesosoma with a large, spine-like posterior pronotal tubercle and a large anterior mesonotal tubercle; mesonotal protuberances spine like; and mesosoma with squamate hairs (Mackay and Baena 1993).
Distribution. Cyphomyrmex castagnei is only known from Panama and the Pacific coast of Colombia (Fig. 1B) (MacKay and Baena 1993; Fernández et al. 1996; Fernández and Sendoya 2004). 
(20) Cyphomyrmex cornutus Kempf, 1968 Figure $5 \mathrm{G}, \mathrm{H}$

Material examined. COLOMBIA: Chocó $・ 7$ workers; Lloró; $05^{\circ} 30^{\prime} 00^{\prime \prime} \mathrm{N}, 076^{\circ} 31^{\prime} 00^{\prime \prime} \mathrm{W}$; alt. $90 \mathrm{~m}$; 25 Oct. 2000; J. Neita leg.; UNAB 545, MEFLG 6936.

Identification. Cephalic and mesonotal protuberances horn-like; preocular carina curves above eye; frontal lobes broadly expanded, semicircular; pronotum without median paired denticles, humeral prominence weakly developed and postero-lateral tubercle more developed, antero-ventral corner rectangular; and mesonotum with anterior pair of very prominent spines and posterior pair of very low denticles (Kempf 1968).

Distribution. Cyphomyrmex cornutus is widely distributed in Central and South America. It has been recorded from Veracruz state in Mexico, south to Costa Rica, Colombia, Ecuador, French Guiana, and the Brazilian states of Acre and Bahia. This ample and somewhat disjunct distribution suggests that $C$. cornutus might have a continuous distribution throughout Central America and northern South America (Adams and Longino 2007; Miranda et al. 2012; Vergara-Navarro and Serna 2013; Santos et al. 2017).

\section{(21) Cyphomyrmex costatus Mann, 1922}

Figure 6A, B

Material examined. COLOMBIA: Antioquia 4 gynes; Amalf; $06^{\circ} 59^{\prime} 01^{\prime \prime} \mathrm{N}, 075^{\circ} 22^{\prime} 06^{\prime \prime} \mathrm{W}$; alt. $975 \mathrm{~m}$; 30 Jul. 1997; F. Serna leg.; MEFLG 40837; UNAB 553, 3461, 4749; Tolima - 2 workers; Guamo; $04^{\circ} 02^{\prime} 05^{\prime \prime} \mathrm{N}$, $074^{\circ} 58^{\prime 2} 25^{\prime \prime}$ W; alt. 323 m; 19 Aug. 2003; F. Serna, E. Díaz leg.; UNAB 4749, 3461.

Identification. Disc of first gastral tergum with strong longitudinal ridges on each side of midline; mid-pronotal tubercles absent; and posterodorsal margin of petiole neither drawn out nor bidentate (Kempf 1965).

Distribution. Cyphomyrmex costatus is continuously distributed throughout Central and northern South America, from the states of Oaxaca and Veracruz in Mexico, to the states of Amazonas and Pará in Brazil (Harada and Ketelhut 2009; Bezděčková et al. 2015).

\section{(22) Cyphomyrmex hamulatus Weber, 1938}

Figure 6C, D

Material examined. COLOMBIA: Antioquia 12 workers; Amalfi; $06^{\circ} 54^{\prime} 11^{\prime \prime} \mathrm{N}, 075^{\circ} 05^{\prime} 26.7^{\prime \prime} \mathrm{W}$; alt. $1010 \mathrm{~m}$; 19 Dec. 1999; F. Serna leg.; UNAB 4750; Antioquia • 1 worker; Amalfi; $06^{\circ} 46^{\prime} 31^{\prime \prime} \mathrm{N}, 075^{\circ} 05^{\prime} 33^{\prime \prime} \mathrm{W}$; alt. 1010 m; Jul-Oct. 1997; F. Serna leg.; UNAB 4750; Antioquia • 2 workers; Amalfi; $06^{\circ} 45^{\prime} 57.6^{\prime \prime} \mathrm{N}, 075^{\circ} 06^{\prime} 25.02^{\prime \prime} \mathrm{W}$; alt. 955 m; Sep. 2006; M. A. Vanegas leg.; MEFLG 11080; Antioquia - 1 worker; Amalfi; $06^{\circ} 47^{\prime} 57.82^{\prime \prime} \mathrm{N}$, $075^{\circ} 07^{\prime} 18.96^{\prime \prime} \mathrm{W}$; alt. $1077 \mathrm{~m}$; Nov. 2006; M. A. Vanegas leg.; MEFLG 11081; Antioquia 5 gynes, 11 workers; Amalfi; $06^{\circ} 47^{\prime} 43^{\prime \prime} \mathrm{N}, 075^{\circ} 07^{\prime} 56^{\prime \prime} \mathrm{W}$; alt. $1550 \mathrm{~m}$; 27 Oct. 1997; F. Serna leg.; MEFLG 5843; Antioquia • 10 workers; Amalfi; $06^{\circ} 46^{\prime} 19.2^{\prime \prime} \mathrm{N}, 075^{\circ} 05^{\prime} 26.44^{\prime \prime} \mathrm{W}$; alt. $1550 \mathrm{~m}$;
30 Jul. 1997; F. Serna leg.; MEFLG 5843; Caquetá • 1 worker; El Doncello; $01^{\circ} 40^{\prime} 48^{\prime \prime} \mathrm{N}, 075^{\circ} 17^{\prime} 06^{\prime \prime} \mathrm{W}$; alt. 500 m; 16 Nov. 2013; F. Serna leg.; UNAB 4368; Caquetá - 3 workers; Florencia; $01^{\circ} 25^{\prime} 34.7^{\prime \prime} \mathrm{N}, 075^{\circ} 30^{\prime} 59^{\prime \prime} \mathrm{W}$; alt. 267 m; Sep. 2017; D. Mera-Rodríguez leg.; UNAB 4750; Caquetá - 2 workers; Florencia; $01^{\circ} 30^{\prime} 9.99^{\prime \prime} \mathrm{N}$, $075^{\circ} 36^{\prime} 23^{\prime \prime} \mathrm{W}$; alt. $250 \mathrm{~m}$; 28 Sep. 2016; UNAB members leg.; UNAB 4369; Sucre - 2 workers; Ovejas; $09^{\circ} 32^{\prime} 4.1^{\prime} 00^{\prime \prime} \mathrm{N}, 075^{\circ} 13^{\prime} 18.5^{\prime \prime} \mathrm{W}$; alt. $277 \mathrm{~m}$; 4 Feb. 2016; H. Cadena leg.; MEFLG 40890-408901.

Identification. Frontal carina quite sinuous; all carina very sharp and somewhat foliaceous; promesonotal tubercles acutely pointed; hind femur angulates at basal third, posteroventral border with a narrow foliaceous crest; postpetiole with a shallow mid-dorsal impression, hairs on head and gaster recurvate or hook-like, not appressed; and tubercles on mesosoma sharply pointed (Kempf 1965).

Distribution. Cyphomyrmex hamulatus is distributed throughout Bolivia, Brazil (Amazonas and Pará), Colombia, Costa Rica, Peru, and Venezuela (Brandão 1991; Snelling and Longino 1992; Fernández and Sendoya 2004).

\section{(23) Cyphomyrmex laevigatus Weber, 1938}

Figure 6E, F

Material examined. COLOMBIA: Caquetá • 1 worker; Florencia; $01^{\circ} 30^{\prime} 9.99^{\prime \prime} \mathrm{N}, 075^{\circ} 36^{\prime} 22^{\prime \prime} \mathrm{W}$; alt. $250 \mathrm{~m}$; 28 Sep. 2016; UNAB members leg.; UNAB 4367.

Identification. Mesosoma completely unarmed, propodeum in profile evenly rounded; and petiolar node in dorsal view strikingly transverse, thrice as broad as long (Kempf 1965).

Distribution. Cyphomyrmex laevigatus is widely distributed in northern South America, including Bolivia, northern Brazil (Amazonas, Maranhão, Mato Grosso, Pará, and Rondônia), Colombia, Ecuador, Paraguay, Perú and Venezuela (Mayhé-Nuñes and Jaffé 1998; Souza et al. 2018).

\section{(24) Cyphomyrmex major (Forel, 1901)}

Figure $6 \mathrm{G}, \mathrm{H}$

Material examined. COLOMBIA: Antioquia 3 gynes, 4 workers; Turbo; $08^{\circ} 05^{\prime} 53^{\prime \prime} \mathrm{N}, 076^{\circ} 43^{\prime} 54^{\prime \prime} \mathrm{W}$; alt. $1 \mathrm{~m} ; 15$ Sep. 2004; O. Munar leg.; UNAB 2353; Santander • 4 gynes, 4 workers; Santa Helena del Opon; $06^{\circ} 20^{\prime} 00^{\prime \prime} \mathrm{N}$, $073^{\circ} 36^{\prime} 00^{\prime \prime} \mathrm{W}$; alt. $1426 \mathrm{~m}$; Apr. 2012; A. Ardila leg.; UNAB 2526.

Identification. Mid-pronotal tubercle paired; cephalic posterolateral corner produced as broad lobe, not spinelike; frontal carina extending onto top lobe joining posterior carina; and anterior mesonotal tubercle high and obtuse (Snelling and Longino 1992).

Distribution. Cyphomyrmex major is known from Bolivia, Brazil (Amazonas, Rio Grande do Sul, Paraná, and São Paulo), Colombia, Costa Rica, Nicaragua, Panama, and Venezuela (Kempf 1972; Santos et al. 2017; Fernández and Serna 2019). 


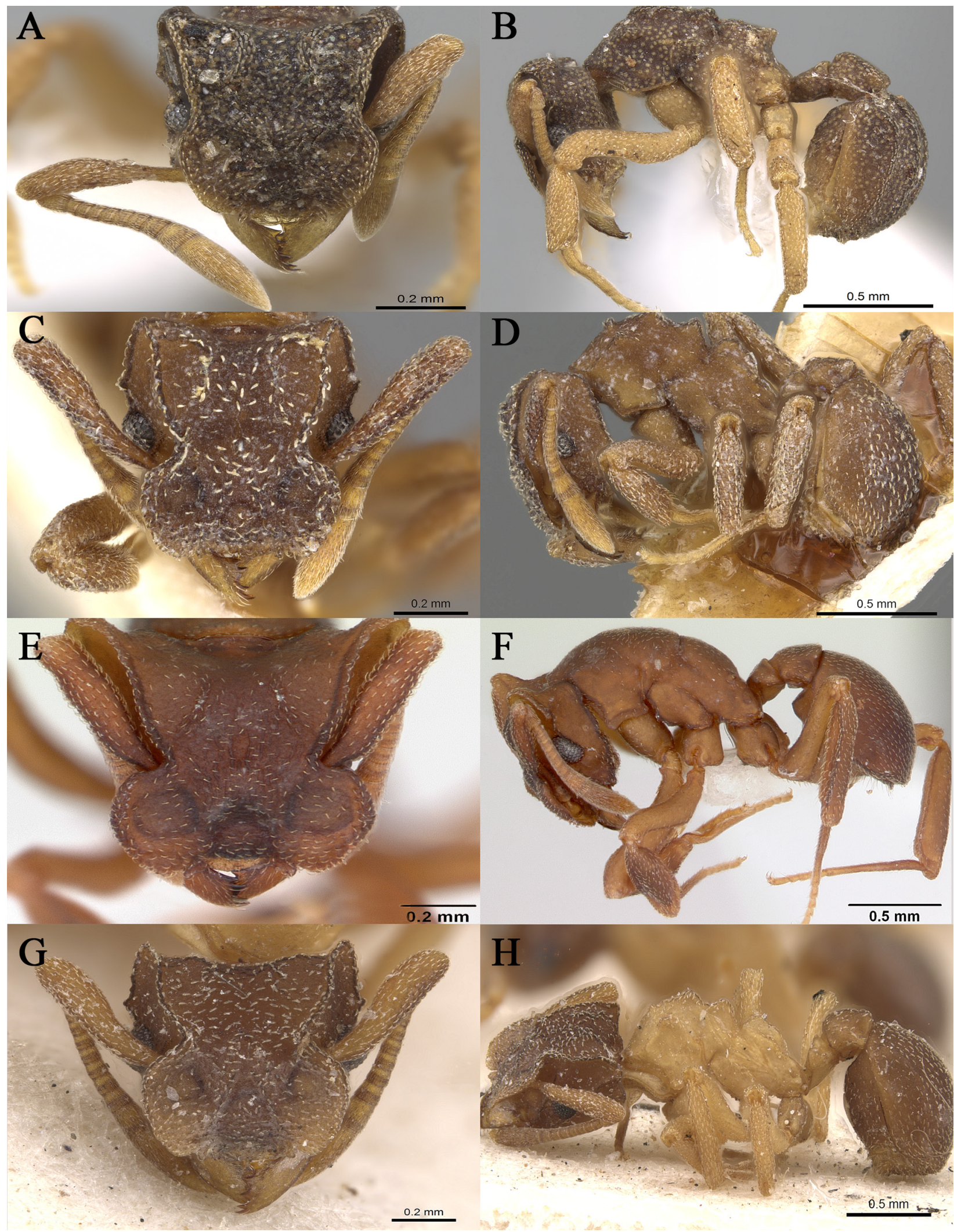

Figure 6. Cyphomyrmex species encountered in Colombia. A, B. Cyphomyrmex costatus, CASENT0281765/ S. Hartman. C, D. Cyphomyrmex hamulatus, CASENT0901673/ R. Perry. E, F. Cyphomyrmex laevigatus, CASENT0173956/ A. Nobile. G, H. Cyphomyrmex major, CASENT0909379/ W. Ericson. Photographs obtained from the Antweb database (http://www.antweb.org).

\section{(25) Cyphomyrmex minutus Mayr, 1862}

Figure $7 \mathrm{~A}, \mathrm{~B}$

Material examined. COLOMBIA: Amazonas $\bullet 1$ worker; La Pedrera; $01^{\circ} 35^{\prime} 00^{\prime \prime} \mathrm{S}, 069^{\circ} 28^{\prime} 00^{\prime \prime} \mathrm{W}$; alt. $87 \mathrm{~m} ; 29$
Apr.-7 May 2001; A. Sabogal leg.; UNAB 4755; Antioquia - 5 workers; Amalfi; $06^{\circ} 46^{\prime} 31^{\prime \prime} \mathrm{N}, 075^{\circ} 05^{\prime} 33^{\prime \prime} \mathrm{W}$; alt. 1010 m; 08 May 1998; F. Serna leg.; UNAB 4751, 4753; Antioquia - 13 workers; Amalfi; 06 ${ }^{\circ} 47^{\prime} 43^{\prime \prime} \mathrm{N}$, $075^{\circ} 07^{\prime} 56^{\prime \prime}$ W; alt. $1550 \mathrm{~m} ; 27$ Oct. 1997; F. Serna leg.; 
MEFLG 5843; Antioquia • 4 workers; Amalfi; 06 $47^{\prime}$ $43^{\prime \prime} \mathrm{N}, 075^{\circ} 07^{\prime} 56^{\prime \prime} \mathrm{W}$; alt. $1550 \mathrm{~m}$; 30 Jul. 1997; F. Serna leg.; MEFLG 5843; Antioquia • 2 workers; Medellín; $06^{\circ} 15^{\prime} 44.4^{\prime \prime} \mathrm{N}, 075^{\circ} 34^{\prime} 33.4^{\prime \prime} \mathrm{W}$; alt. $1460 \mathrm{~m}$; May 1999; MELFG members leg.; MEFLG 5890; Antioquia - 4 workers; Santafé de Antioquia; 06 $33^{\prime} 41^{\prime \prime} \mathrm{N}, 075^{\circ}$ 49'54"W; alt. 600 m; 06 Oct. 2000; A. Miranda leg.; UNAB 4752; Caquetá • 1 worker; Florencia; 01³0'4"N, 075³9'42"W; alt. $280 \mathrm{~m}$; Sep. 2017; UNAB members leg.; UNAB 4751; Caquetá • 34 workers; Florencia; 01²5'34"N, 075³0'59"W; alt. 267 m; Sep. 2017; D. Mera-Rodríguez leg.; UNAB 4751; Caquetá • 4 workers; Florencia; 01³0'4"N, 075³9'42"W; alt. 280 m; Sep. 2017; D. Mera-Rodríguez leg.; UNAB 4751, 4753; Santander • 1 gyne, 4 workers; San Vicente del Chucurí; 06 $54^{\prime} 5^{\prime \prime} \mathrm{N}$, 07344'8"W; alt. 102 m; F. Serna leg.; UNAB 4751.

Identification. Small individuals; head width $0.56 \mathrm{~mm}$ or less; hairs on disc of gaster closely appressed and mostly separated by more than their own lengths; and median basal groove of gaster short and usually indistinct (Snelling and Longino 1992).

Distribution. Cyphomyrmex minutus is one of the most widely distributed species of fungus-growing ants. It has been recorded from southern United States of America (Arizona, Texas, and Florida) all the way south to Argentina (Misiones) (Snelling and Longino 1992; Deyrup 2003, 2016; Fernández and Sendoya 2004; Salinas 2010; Demétrio et al. 2017). It is possible that $C$. minutus is a complex of cryptic species, but this hypothesis needs to be tested with a comprehensive sampling of populations from across the Americas.

\section{(26) Cyphomyrmex rimosus (Spinola, 1851)} Figure 7C, D

Material examined. COLOMBIA: Amazonas - 1 worker; La Pedrera; 01³5'00"S, $069^{\circ} 28^{\prime} 00^{\prime \prime} \mathrm{W}$; alt. 87 m; 29 Apr.-7 May 2001; A. Sabogal leg.; UNAB 4755; Antioquia - 5 workers; Amalfi; 06 $47^{\prime} 43^{\prime \prime} \mathrm{N}, 075^{\circ}$ 07'56"W; alt. 1550 m; 27 Oct. 1997; F. Serna leg.; MEFLG 5843; Caquetá - 4 workers; Florencia; 01³0'4"N, 075³9'42"W; alt. 280 m; Sep. 2017; D. Cubillos leg.; UNAB 4754; Caquetá - 1 worker; La Montanita; $01^{\circ} 28^{\prime} 25^{\prime \prime} \mathrm{N}, 075^{\circ} 32^{\prime} 51^{\prime \prime} \mathrm{W}$; alt. $612 \mathrm{~m}$; 27 Dec. 2015; D. Meneses leg.; UNAB 3900; Cundinamarca • 1 worker; Choachi; 04³1'52"N, 07355'33"W; alt. 1927 m; 04 Nov. 2013; M. Forero leg.; UNAB 4754; Huila • 1 worker; El Pital; 02²0'53"N, 07547'55"W; alt. $921 \mathrm{~m}$; 02 Nov. 2009; D. Briceno leg.; UNAB 4754.

Identification. Large individuals, head width more than $0.62 \mathrm{~mm}$; hairs of gaster coarse, not fully appressed and mostly separated by less than their own lengths; and basal groove of gaster distinct and more than twice as long as wide (Snelling and Longino 1992).

Distribution. Similar to C. minutus, C. rimosus is also widely distributed in the Americas, its distribution extends from southern United States of America, throughout Central and South America to Argentina (Buenos
Aires and Mendoza) (Kempf 1972; Fernández and Sendoya 2004). A thorough taxonomic study needs to test whether C. rimosus is a single biological species, or whether it is a complex of cryptic species.

Mycocepurus Forel, 1893

\section{(27) Mycocepurus smithii (Forel, 1893)}

Figure 7E, F

Material examined. COLOMBIA: Antioquia • 1 worker; Amalfi; $06^{\circ} 45^{\prime} 42.23^{\prime \prime} \mathrm{N}, 075^{\circ} 05^{\prime} 31.57^{\prime \prime} \mathrm{W}$; alt. $983 \mathrm{~m}$; Sep. 2006; M.A. Vanegas leg.; MEFLG 11088; Antioquia - 1 worker; Santafé de Antioquia; 06 $33^{\prime} 41^{\prime \prime} \mathrm{N}, 075^{\circ}$ 49'54"W; alt. 600 m; 06 Oct. 2000; E. Vergara, F. Serna leg.; UNAB 4756; Antioquia - 2 workers; Turbo; 08 $05^{\prime} 53^{\prime \prime} \mathrm{N}, 076^{\circ} 43^{\prime} 54^{\prime \prime} \mathrm{W}$; alt. $1 \mathrm{~m}$; 15 Sep. 2004; O. Munar leg.; UNAB 4756; Caquetá • 2 workers; Florencia; $01^{\circ} 37^{\prime} 11^{\prime \prime} \mathrm{N}, 075^{\circ} 36^{\prime} 14^{\prime \prime} \mathrm{W}$; alt. 300 m; 31 Mar. 2016; F. Serna leg.; UNAB 4357; Caquetá • 5 workers; Florencia; $01^{\circ}$ $30^{\prime} 4^{\prime \prime} \mathrm{N}, 075^{\circ} 39^{\prime} 42^{\prime \prime} \mathrm{W}$; alt. 280 m; Sep. 2017; UNAB leg.; UNAB 4756.

Identification. Pronotum with a pair of anterior pronotal spines, which define the anterior border of a crown of spines on the promesonotum; promesonotal disc lacking spines; lateral pronotal spines high and sharp; and propodeal spines usually straight (Mackay et al. 2004).

Distribution. Mycocepurus smithii is widely distributed throughout Central and South America, extending from Mexico all the way south to Argentina including many Caribbean islands (Kempf 1972; Fernández et al. 1996; Mackay et al. 2004; Rabeling et al. 2011; Franco and Feitosa 2018).

\section{Myrmicocrypta Smith, 1860}

\section{(28) Myrmicocrypta urichi Weber, 1937}

Figure $7 \mathrm{G}, \mathrm{H}$

Material examined. COLOMBIA: Antioquia • 1 worker; Santafé de Antioquia; 06 33'41"N, 075 49'54"W; alt. 600 m; Nov. 2017; D. Mera-Rodríguez leg.; UNAB 4758;

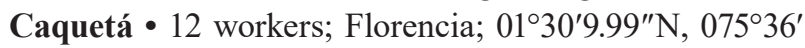
23"W; alt. 250 m; 28 Sep. 2016; UNAB members leg.; UNAB 4358; Caquetá - 12 workers; Florencia; 01³7' $11^{\prime \prime} \mathrm{N}, 075^{\circ} 36^{\prime} 14^{\prime \prime} \mathrm{W}$; alt. 300 m; 31 Mar. 2016; F. Serna leg.; UNAB 4358; Caquetá - 3 workers; Florencia; $01^{\circ} 25^{\prime} 34^{\prime \prime} \mathrm{N}, 075^{\circ} 30^{\prime} 59^{\prime \prime} \mathrm{W}$; alt. 267 m; Sep. 2017; D. MeraRodríguez leg.; UNAB 4758; Caquetá • 15 workers; Florencia; $01^{\circ} 30^{\prime} 4^{\prime \prime} \mathrm{N}, 075^{\circ} 39^{\prime} 42^{\prime \prime} \mathrm{W}$; alt. $280 \mathrm{~m}$; Sep. 2017; UNAB members leg.; UNAB 4758; Caquetá • 6 workers; Florencia; 01²5'34"N, 075³0'59"W; alt. 267 m; Sep. 2017; D. Cubillos leg.; UNAB 4758; Caquetá • 17 workers; Florencia; $01^{\circ} 30^{\prime} 4^{\prime \prime} \mathrm{N}, 075^{\circ} 39^{\prime} 42^{\prime \prime} \mathrm{W}$; alt. 280 m; Sep. 2017; UNAB members leg.; UNAB 4758; Caquetá • 25 workers; Florencia; 01³0'4"N, 075³9'42"W; alt. 280 m; Sep. 2017; D. Pantoja leg.; UNAB 4758; Caquetá • 18 workers; Florencia; 01²5'34"N, 075³0'59"W; alt. 267 m; Sep. 2017; F. Serna, D. Cubillos leg.; UNAB 4758; Caquetá • 3 workers; Florencia; $01^{\circ} 30^{\prime} 4^{\prime \prime} \mathrm{N}, 075^{\circ} 39^{\prime} 42^{\prime \prime} \mathrm{W}$; alt. $280 \mathrm{~m}$; Sep. 


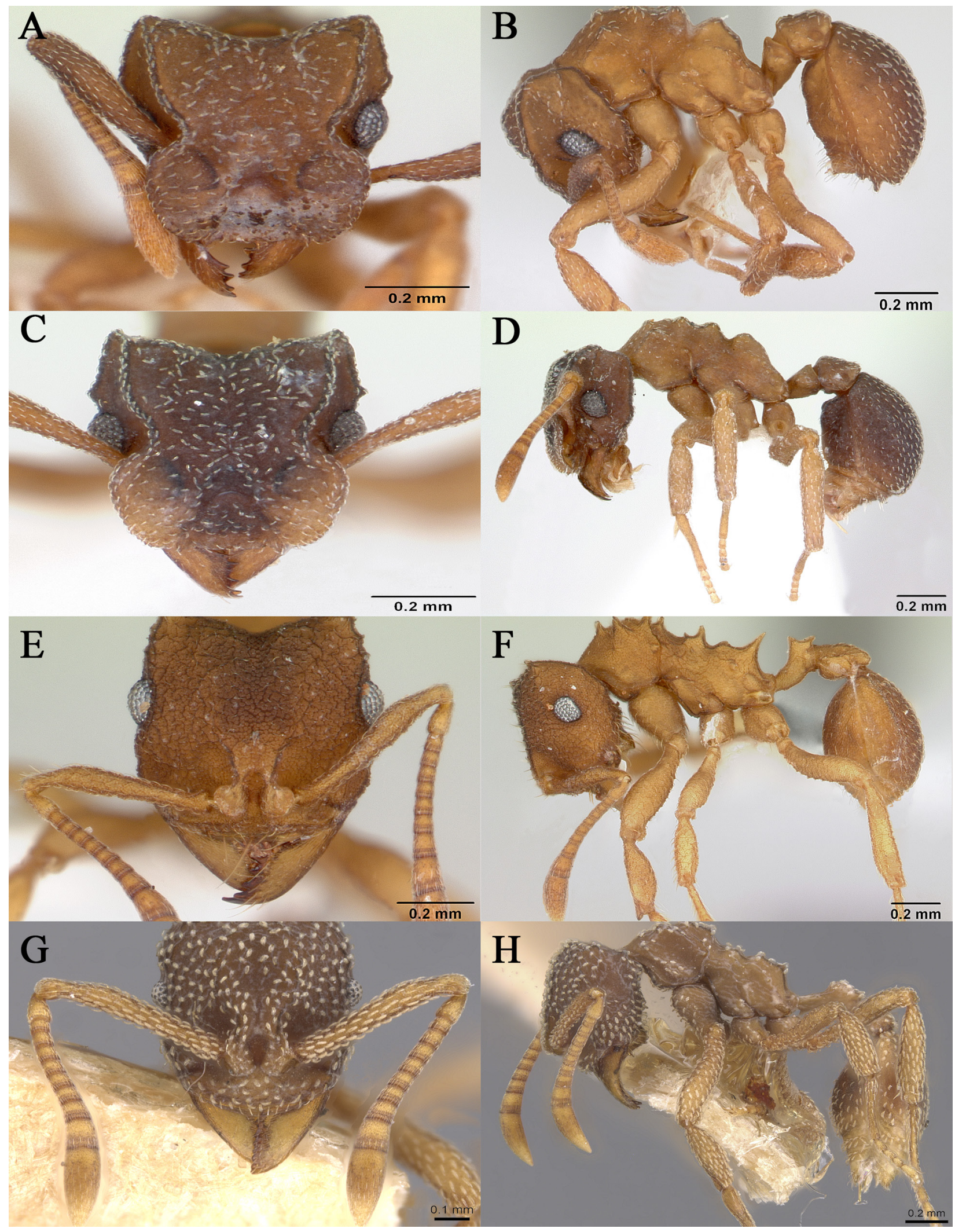

Figure 7. Cyphomyrmex, Mycocepurus, and Myrmicocrypta species encountered in Colombia. A, B. Cyphomyrmex minutus, CASENT0173957. C, D. Cyphomyrmex rimosus, CASENT0173243. E, F. Mycocepurus smithii, CASENT0173989. G, H. Myrmicocrypta urichi, CASENT0912513. Photographs taken by A. Nobile (A-F) and W. Ericson $(\mathbf{G}, \mathbf{H})$ obtained from the Antweb database (http://www.antweb.org).

2017; F. Serna, D. Cubillos Leg.; UNAB 4758; Caquetá • 9 workers; Florencia; 01²5'34"N, 075³0'59"W; alt. 267 m; Sep. 2017; D. Mera-Rodríguez leg.; UNAB 4758; Caquetá • 6 workers; Florencia; $01^{\circ} 30^{\prime} 4^{\prime \prime} \mathrm{N}, 075^{\circ} 39^{\prime} 42^{\prime \prime} \mathrm{W}$; alt. 280 m; Sep. 2017; D. Mera-Rodríguez leg.; UNAB 4758.
Identification. Dark brown ants; body covered by scales; apical mandibular tooth in anterior view as long as preapical tooth; clypeus, front and frontal lobes densely covered with scales; postpetiole in dorsal view shaped as longitudinal half of an ellipse; and postpetiole $2.5 \times$ 
broader than long (Sosa-Calvo 2015).

Distribution. Myrmicocrypta urichi occurs in Colombia, the Lesser Antilles, and Venezuela (Kempf 1972; Sosa-Calvo 2015).

\section{(29) Myrmicocrypta sp. 1}

Material examined. COLOMBIA: Cesar • 2 workers; Guacoche; $10^{\circ} 40^{\prime} 55^{\prime \prime} \mathrm{N}, 073^{\circ} 17^{\prime} 29^{\prime \prime} \mathrm{W}$; alt. 250-150 m; Jan. 2011; Y. Dominguez leg.; UNAB 4757.

Comment. This is a new species only known from two workers and will be described in a forthcoming taxonomic study. This species corresponds to species Myrmicocrypta ca. ednaella in Sosa-Calvo (2015).

Sericomyrmex Mayr, 1865

\section{(30) Sericomyrmex amabilis Wheeler, 1925} Figure 8A, B

Material examined. COLOMBIA: Antioquia • 4 workers; Santafé de Antioquia; 06 $33^{\prime} 41^{\prime \prime} \mathrm{N}, 075^{\circ} 49^{\prime} 54^{\prime \prime} \mathrm{W}$; alt. 600 m; 06 Oct. 2000; E. Vergara, F. Serna leg.; UNAB 4759; Amazonas • 3 workers; La Pedrera; 01³5'00"S, $069^{\circ} 30^{\prime} 00^{\prime \prime} \mathrm{W}$; alt. $87 \mathrm{~m}$; 12-21 Apr. 2001; A. Sabogal leg.; UNAB 4759; Caquetá - 4 workers; Florencia;

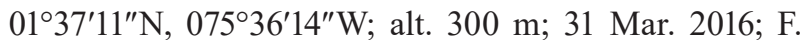
Serna leg.; UNAB 4364; Caquetá • 3 workers; Florencia; 01'30'4"N, 075³9'42"W; alt. 280 m; Sep. 2017; UNAB members leg.; UNAB 4759; Caquetá • 1 worker; Florencia; 01³0'4"N, 075³9'42"W; alt. 280 m; Sep. 2017; D. Cubillos leg.; UNAB 4759; Chocó • 1 worker; Lloró; $05^{\circ} 29^{\prime} 00^{\prime \prime} \mathrm{N}, 076^{\circ} 32^{\prime} 00^{\prime \prime} \mathrm{W}$; alt. 90 m; 26 Nov. 1998; J. Neita, M. Renteria leg.; MEFLG 5902; Chocó • 6 workers; Unguía; $08^{\circ} 02^{\prime} 00^{\prime \prime} \mathrm{N}, 077^{\circ} 05^{\prime} 00^{\prime \prime} \mathrm{W}$; alt. $7 \mathrm{~m}$; Jan. 2002; F. Serna leg.; UNAB 4759; Meta • 1 worker;

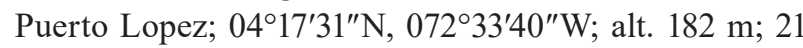
Apr. 2011; M. Montanez leg.; UNAB 4759; Tolima • 1

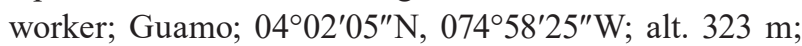
18 Aug. 2003; F. Serna, E. Díaz leg.; UNAB 4759; Tolima • 1 worker; Icononzo; $04^{\circ} 10^{\prime} 32^{\prime \prime} \mathrm{N}, 074^{\circ} 32^{\prime} 00.5^{\prime \prime} \mathrm{W}$; alt. 480 m; 1 Jul. 1997; C. Sarmiento leg.; MEFLG 5381.

Identification. Medium-sized species; mandible usually striate; frontal carina complete; frontal lobe triangular; eye almost flat, without white layer; posterior cephalic margin with abrupt to gradual emargination; mesosomal tubercles from low and obtuse to well-developed; and gaster with lateral carina strongly developed, dorsal carina from weak to well-developed (Ješovnik and Schultz 2017).

Distribution. Sericomyrmex amabilis is present in Central America and occurs from San Luis Potosí in Mexico to Panama. In South America, S. amabilis has been reported from Colombia, Ecuador, and Venezuela (Wheeler 1925; Ješovnik and Schultz 2017).

\section{(31) Sericomyrmex bondari Borgmeier, 1937} Figure 8C, D

Material examined. COLOMBIA: Antioquia - 1

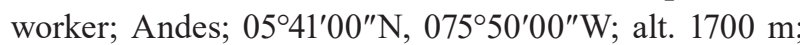

09 Mar. 2017; M. Ramirez leg.; UNAB 4760; Amazonas - 1 worker; La Pedrera; $01^{\circ} 35^{\prime} 00^{\prime \prime} \mathrm{S}, 069^{\circ} 28^{\prime} 00^{\prime \prime} \mathrm{W}$; alt. 87 m; 29 Apr.-7 May 2001; A. Sabogal leg.; UNAB 4760; Caquetá - 2 workers; Florencia; 01³0'9.99"N, $075^{\circ} 36^{\prime} 23^{\prime \prime} \mathrm{W}$; alt. $250 \mathrm{~m}$; 21 Sep. 2016; F. Serna leg.; UNAB 363; Putumayo • 1 worker; Mocoa; $01^{\circ} 08^{\prime} 00^{\prime \prime} \mathrm{N}$, 076 38'00"W; alt. 500 m; 11-13 Jun. 2002; UNAB members leg.; UNAB 4773; Vaupés - 1 worker; Taraira; $01^{\circ} 04^{\prime} 00^{\prime \prime} \mathrm{S}, 069^{\circ} 31^{\prime} 00^{\prime \prime} \mathrm{W}$; alt. $85 \mathrm{~m}$; 14-20 May 2001; A. Sabogal leg.; UNAB 4760.

Identification. Large species; hairs thick and dark; posterior cephalic emargination deep, gradually impressed; posterior cephalic corner acute to rounded; mandible dorsally smooth; frontal lobe triangular; mesosomal tubercles distinct, sometimes relatively sharp; and gaster with lateral carina weakly to moderately developed, and dorsal carina absent (Ješovnik and Schultz 2017).

Distribution. Sericomyrmex bondari is widely distributed throughout South America and has been recorded in Bolivia, the Brazilian states of Amazonas, Bahia, Ceará, Espírito Santo, Mato Grosso, Minas Gerais, Pernambuco, Rio de Janeiro, and Rondônia, as well as in Colombia, Ecuador, French Guiana, Guyana, Peru, Suriname, and Venezuela (Borgmeier 1937; Ješovnik and Schultz 2017).

\section{(32) Sericomyrmex mayri Forel, 1912}

Figure 8E, F

Material examined material. COLOMBIA: Antioquia - 1 worker; Amalfi; 06 $46^{\prime} 19.2^{\prime \prime} \mathrm{N}, 075^{\circ} 05^{\prime} 24.4^{\prime \prime} \mathrm{W}$; alt. 1550 m; 1 Jul. 1998; F. Serna leg.; MEFLG 5901; Antioquia • 3 workers; Amalfi; 06 $45^{\prime} 57.6^{\prime \prime} \mathrm{N}, 075^{\circ} 06^{\prime} 25.02^{\prime \prime} \mathrm{W}$; alt. 955 m; Sep. 2006; M.A. Vanegas leg.; MEFLG 5901; Antioquia - 1 worker; Amalfi; 06 $47^{\prime} 48.92^{\prime \prime} \mathrm{N}, 075^{\circ}$ $06^{\prime} 55.56^{\prime \prime} \mathrm{W}$; alt. 1113 m; 2 Oct. 1998; F. Serna leg.; MEFLG 5901; Antioquia • 3 workers; Amalfi; $06^{\circ} 47^{\prime} 48.92^{\prime \prime} \mathrm{N}$, $075^{\circ} 06^{\prime} 55.56^{\prime \prime} \mathrm{W}$; alt. 1113 m; 1997; F. Serna leg.; MEFLG 5901; Antioquia • 1 worker; San Carlos; 06²11'29"N, 07459'43"W; alt. 1036 m; 05 Jan. 2007; N. Vergara, C. Gómez leg.; UNAB 4774; Sucre - 3 workers; Ovejas; 09'32'4.1"N, 075'13'18.5"W; alt. 277 m; 4 Feb. 2016; H. Cadena leg.; MEFLG 40894, 40893, 40892.

Identification. Large species; head broad; frontal lobe narrow; mandible usually striate; frontal carina often incomplete; eye flat to mildly convex; posterior cephalic margin shallow, abruptly to gradually impressed; posterior cephalic corner usually angled; mesosomal tubercles low and obtuse; and gaster with lateral carina well-developed, dorsal carina absent or faint (Ješovnik and Schultz 2017).

Distribution. Sericomyrmex mayri has been recorded from Bolivia, the northern states of Brazil, Colombia, Ecuador, Peru, and Venezuela. In Central America, $S$. mayri is only known in Panama (Forel 1912; Ješovnik and Schultz 2017). 


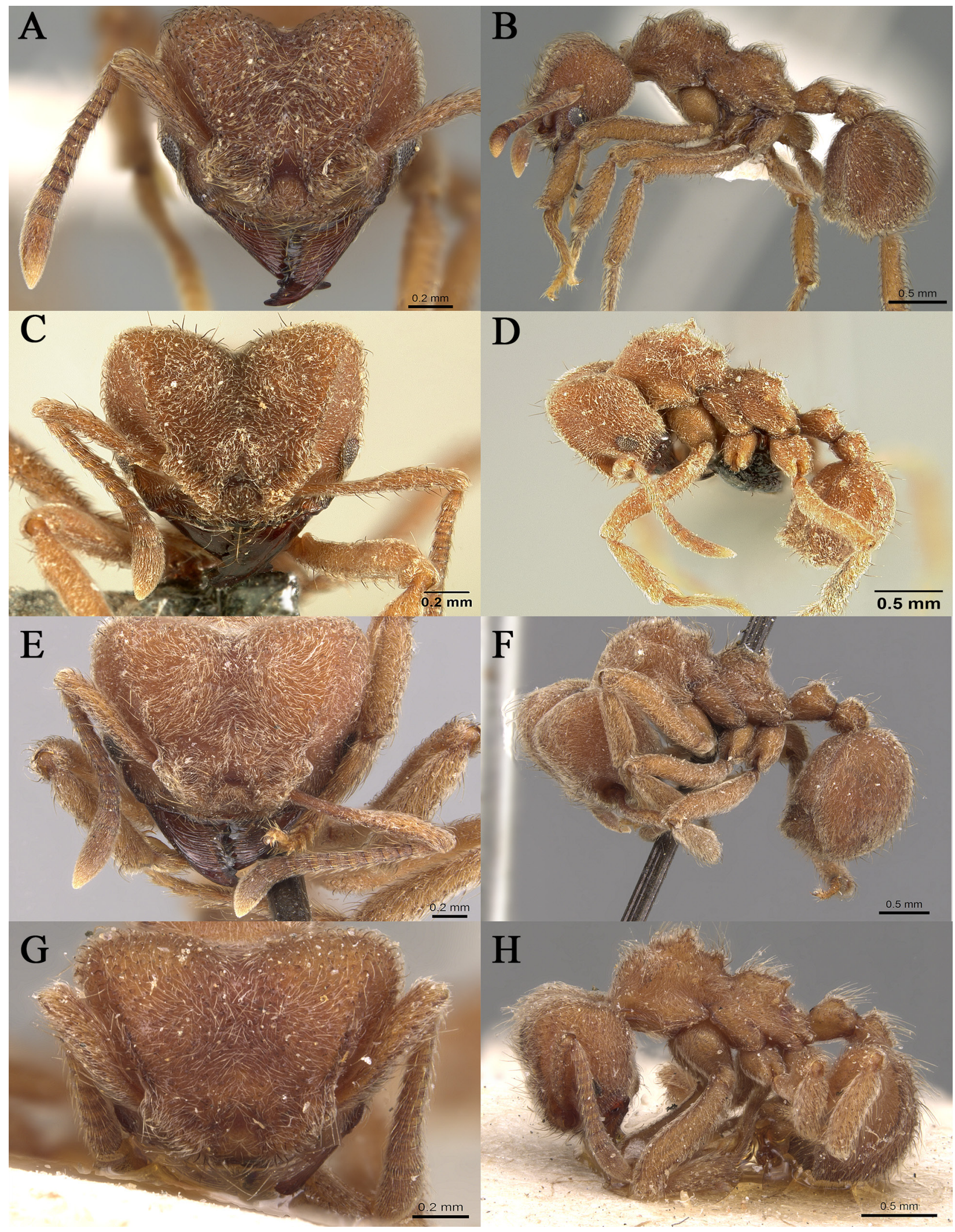

Figure 8. Sericomyrmex species encountered in Colombia. A, B. Sericomyrmex amabilis, CASENT0922156/ M. Esposito. C, D. Sericomyrmex bondari, USNMENT01125823/ A. Ješovnik. E, F. Sericomyrmex mayri, CASENT0909370/ W. Ericson. G, H. Sericomyrmex saussurei, USNMENT00445513/ W. Ericson. Photographs obtained from the Antweb database (http://www.antweb.org).

(33) Sericomyrmex saussurei Emery, 1894

Figure $8 \mathrm{G}, \mathrm{H}$

Material examined. COLOMBIA: Amazonas • 2 workers; La Pedrera; $01^{\circ} 35^{\prime} 00^{\prime \prime} \mathrm{S}, 069^{\circ} 30^{\prime} 00^{\prime \prime} \mathrm{W}$; alt. $87 \mathrm{~m}$;
12-21 Apr. 2001; A. Sabogal leg.; UNAB 4761; Santander - 1 worker; Santa Helena del Opón; 06 $20^{\prime}$ $00^{\prime \prime} \mathrm{N}, 073^{\circ} 36^{\prime} 00^{\prime \prime} \mathrm{W}$; alt. 1426 m; 01 Apr. 2012; A. Ardila leg.; UNAB 2528. 
Identification. Medium-sized species; mandible usually striate; frontal carina complete; frontal lobe triangular; eye convex, moderately protruding from sides of head, covered with thick white layer; posterior cephalic emargination abruptly to gradually impressed, mesosomal tubercles from low and obtuse to well developed; and gaster with lateral carina well developed, and dorsal carina weak to well developed (Ješovnik and Schultz 2017).

Distribution. Sericomyrmex saussurei is widely distributed throughout the Amazon basin (Emery 1894; Kempf 1972; Ješovnik and Schultz 2017).

Mycetomoellerius Solomon et al., 2019

\section{(34) Mycetomoellerius jamaicensis (Andre, 1893) Figure 9A, B}

Material examined. COLOMBIA: Magdalena - 27 workers; Pivijay; $10^{\circ} 27^{\prime} 00^{\prime \prime} \mathrm{N}, 074^{\circ} 36^{\prime} 00^{\prime \prime} \mathrm{W}$; alt. $3 \mathrm{~m}$; Nov. 1985; A. Madrigal leg.; MEFLG 4272; Magdalena - 14 workers; Pivijay; $10^{\circ} 27^{\prime} 00^{\prime \prime} \mathrm{N}, 074^{\circ} 36^{\prime} 00^{\prime \prime} \mathrm{W}$; alt. 3 m; May 1986; A. Madrigal leg.; MEFLG 4822.

Identification. Antennal scapes surpass posterior corners of head by 1-2 times their maximum diameter; clypeus with row of coarse, long hairs on anterior margin; in side view, a few shorter erect hairs often present posterior to the anterior row; preocular carina relatively short, stopping at about $1 / 3$ the distance between eye and posterior corner of head; frontal carina long, reaching back to posterior corner of head; anterolateral promesonotal tooth long, sharply pointed, projecting forward and upwards; propodeal teeth sharply pointed, approximately as long as the distance between their bases; gaster strongly tuberculated, in dorsal view tubercles form four more or less distinct longitudinal ridges on first gastric tergite; and color dark reddish-black or gray-black, appendages and two petiolar segments usually a lighter reddish-brown (Rabeling et al. 2007).

Distribution. Mycetomoellerius jamaicensis was previously recorded from the Caribbean island arc, Florida (USA), and Venezuela (Deyrup 2003, 2016; Jaffé and Lattke 1994; Mayhé-Nuñes and Brandão 2007; Rabeling et al. 2007). Our new record from Magdalena department in northern Colombia extends the distribution along the northern South American mainland (Fig. 1C).

\section{(35) Mycetomoellerius urichii (Forel, 1893)}

Figure 9C, D

Material examined. COLOMBIA: Cesar • 1 worker; Valledupar; $10^{\circ} 23^{\prime} 17^{\prime \prime} \mathrm{N}, 073^{\circ} 14^{\prime} 11^{\prime \prime} \mathrm{W}$; alt. $250 \mathrm{~m}$; Mar. 2011; Y. Dominguez leg.; UNAB.

Identification. Head square, as wide as long; mandibles subopaque smooth and sparsely punctuated at apex, indistinctly denticulate at the terminal border; median pronotal spines tuberculated; lateral pronotal spines slender and with small tubercles; mesonotum with four tuberculated spines; and propodeal spines robust and with small tubercles (Forel 1893; Fernández and Serna 2019).
Distribution. Mycetomoellerius urichii is present in Colombia, French Guiana, Guyana, the Lesser Antilles, Panama, Suriname, Venezuela, and much of Brazil (Bahia, Goiás, Mato Grosso, Mato Grosso do Sul, Minas Gerais, Santa Catarina, São Paulo, Rio de Janeiro, and Tocantins) (Kempf 1972; Fernández and Sendoya 2004).

\section{(36) Mycetomoellerius sp. 1}

Material examined. COLOMBIA: Antioquia • 1 worker; Amalfi; 06 $57^{\prime} 8.02^{\prime \prime} \mathrm{N}, 075^{\circ} 00^{\prime} 48^{\prime \prime} \mathrm{W}$; alt. $1020 \mathrm{~m}$; 27 Oct. 1997; F. Serna leg.; UNAB 5452; Atlántico • 1 worker; Juan de Acosta; $10^{\circ} 46^{\prime} 02^{\prime \prime} \mathrm{N}, 075^{\circ} 02^{\prime} 34^{\prime \prime} \mathrm{W}$; alt. 117-250 m; Feb.-May 2012; J. Castro, S. Royero leg.; UNAB 5452; Chocó • 1 worker; Unión Panamericana; $05^{\circ} 21^{\prime} 37.48^{\prime \prime} \mathrm{N}$, 076³8'45.35"W; alt. 119 m; 08 May 2001; J. Neita leg.; UNAB 5452; Sucre • 1 worker; Colosó; 09²9'44"N, $075^{\circ}$ 23'17"W; alt. 200-700 m; Aug. 2009; G. Mercado leg.; UNAB 5452.

Comment. This Mycetomoellerius species represents a new species and needs to be described in the context of a taxonomic revision.

\section{Paratrachymyrmex Solomon et al., 2019}

\section{(37) Paratrachymyrmex bugnioni (Forel, 1912)}

Figure 9E, F

Material examined. COLOMBIA: Atlántico • 1 worker; Juan de Acosta; $10^{\circ} 46^{\prime} 02^{\prime \prime} \mathrm{N}, 075^{\circ} 02^{\prime} 34^{\prime \prime} \mathrm{W}$; alt. 177-250 m; Aug. 2012; D. Serna leg.; UNAB 4762.

Identification. Frons and vertex strongly longitudinally rugose; apex of antennal scape scarcely surpassing the posterior cephalic corner; constriction of the frontal carina immediately beyond of the frontal lobes is not present; and distance between the frontal carina is half of the maximum width of the head as measured across the eyes (unpublished key by Kempf).

Distribution. Paratrachymyrmex bugnioni has been recorded from Mexico (Jalisco) and Guatemala south to Brazil (Amazonas, Goiás, Maranhão, Minas Gerais, Pará, and Roraima) (Kempf 1972; Fernández and Sendoya 2004; Achury and Suárez 2018; Souza et al. 2018).

\section{(38) Paratrachymyrmex cornetzi (Forel, 1912)}

Figure 9G, H

Material examined. COLOMBIA: Antioquia • 6 workers; Amalfi; 06 $59^{\prime} 30^{\prime \prime} \mathrm{N}, 074^{\circ} 59^{\prime} 54^{\prime \prime} \mathrm{W}$; alt. $1550 \mathrm{~m}$; 30 Jul. 1997; F. Serna leg.; UNAB 4763; Antioquia • 1 worker; Amalfi; $06^{\circ} 51^{\prime} 26^{\prime \prime} \mathrm{N}, 075^{\circ} 05^{\prime} 40^{\prime \prime} \mathrm{W}$; alt. $970 \mathrm{~m}$; 19 Dec. 1999; E. Vergara leg.; UNAB 4763; Antioquia • 11 workers; Amalfi; 06 $59^{\prime} 30^{\prime \prime} \mathrm{N}, 074^{\circ} 59^{\prime} 54^{\prime \prime} \mathrm{W}$; alt. 1550 m; 27 Oct. 1997; F. Serna leg.; UNAB 4763; Antioquia • 1 worker; Amalfi; $06^{\circ} 51^{\prime} 00^{\prime \prime} \mathrm{N}, 075^{\circ} 05^{\prime} 00^{\prime \prime} \mathrm{W}$; alt. $970 \mathrm{~m}$; 30 Jul. 1997; F. Serna leg.; UNAB 4763; Antioquia • 2 workers; Amalfi; 06 $35^{\prime} 54^{\prime \prime} \mathrm{N}, 075^{\circ} 01^{\prime} 17^{\prime \prime} \mathrm{W}$; alt. $1010 \mathrm{~m}$; 30 Jul. 1997; F. Serna leg.; UNAB 4763; Antioquia • 1 worker; Amalfi; $06^{\circ} 51^{\prime} 00^{\prime \prime} \mathrm{N}, 075^{\circ} 05^{\prime} 00^{\prime \prime} \mathrm{W}$; alt. $970 \mathrm{~m}$; 27 Oct. 1997; F. Serna leg.; UNAB 4763; Antioquia • 2 workers; Amalfi; $06^{\circ} 35^{\prime} 54^{\prime \prime} \mathrm{N}, 075^{\circ} 01^{\prime} 17^{\prime \prime} \mathrm{W}$; alt. 1010 


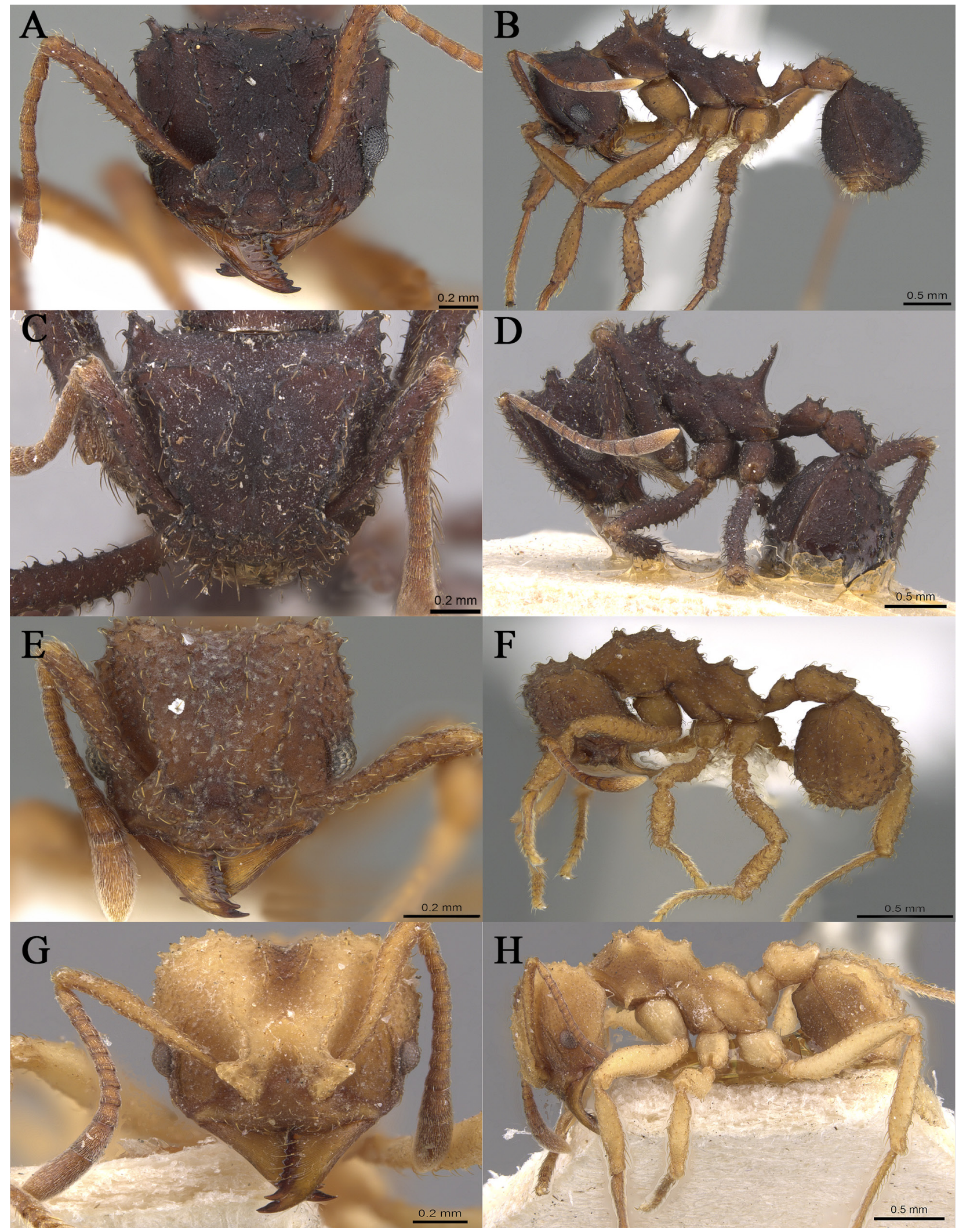

Figure 9. Mycetomoellerius and Paratrachymyrmex species encountered in Colombia. A, B. Mycetomoellerius jamaicensis, CASENT0919971/ M. Esposito. C, D. Mycetomoellerius urichii, CASENT0904990/ W. Ericson. E, F. Paratrachymyrmex bugnioni, CASENT0919968/ M. Esposito. G, H. Paratrachymyrmex cornetzi, CASENT0909389/ W. Ericson. Photographs obtained from the Antweb database (http://www.antweb.org).

m; 08 Oct. 1997; F. Serna leg.; UNAB 4763; Antioquia

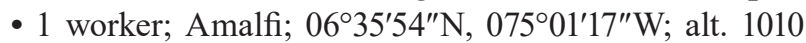
m; 09 Oct. 1997; F. Serna leg.; UNAB 4763; Antioquia - 5 workers; Amalfi; 06 $47^{\prime} 43^{\prime \prime} \mathrm{N}, 075^{\circ} 07^{\prime} 56^{\prime \prime} \mathrm{W}$; alt. 1550 m; 30 Jul. 1997; F. Serna leg.; MEFLG 5918; Antioquia •
3 workers; Amalfi; 06²4'39"N, 07505'22.9"W; alt. 950 m; 27 Oct. 1997; F. Serna leg.; MEFLG 5918; Antioquia - 1 worker; Amalfi; $06^{\circ} 46^{\prime} 39^{\prime \prime} \mathrm{N}, 075^{\circ} 05^{\prime} 22^{\prime \prime} \mathrm{W}$; alt. 1550 m; 30 Jul. 1997; F. Serna leg.; MEFLG 5918; Antioquia - 1 worker; Amalfi; $06^{\circ} 46^{\prime} 19^{\prime \prime} \mathrm{N}, 075^{\circ} 05^{\prime} 26^{\prime \prime} \mathrm{W}$; alt. 1550 
m; 7 Nov. 1998; E. Vergara leg.; MEFLG 5918; Antioquia • 1 gyne; Amalfi; 06²6'19"N, 07505'26.4"W; alt. 1550 m; 8 Oct. 1997; E. Vergara leg.; MEFLG 5918; Antioquia - 1 gyne; Amalfi; 06 $46^{\prime} 19^{\prime \prime} \mathrm{N}, 075^{\circ} 05^{\prime} 26.4^{\prime \prime} \mathrm{W}$; alt. 1550 m; 1 Jul. 1998; E. Vergara leg.; MEFLG 5918; Antioquia - 4 workers; Canyon del Porce; 06 $35^{\prime} 54^{\prime \prime} \mathrm{N}$, 07501'17"W; alt. 1010 m; 28 Oct. 1997; F. Serna leg.; UNAB 4766; Santander • 1 worker; Santa Helena del Opon; $06^{\circ} 20^{\prime} 00^{\prime \prime} \mathrm{N}, 073^{\circ} 36^{\prime} 00^{\prime \prime} \mathrm{W}$; alt. $1426 \mathrm{~m}$; Apr. 2012; A. Ardila leg.; UNAB 2525; Tolima • 1 worker; Guamo; $04^{\circ} 02^{\prime} 05^{\prime \prime} \mathrm{N}, 074^{\circ} 58^{\prime} 25^{\prime \prime} \mathrm{W}$; alt. $323 \mathrm{~m}$; 19 Aug. 2003; F. Serna, E. Díaz leg.; UNAB 4763.

Identification. Frontal lobes triangular; maximum distance between frontal carina is less than the distance between the frontal lobes; and posterior postpetiolar margin in dorsal view emarginate with two prominent tubercles (unpublished key by Kempf).

Distribution. Paratrachymyrmex cornetzi occurs in Mexico (Jalisco and Tabasco), Costa Rica, Guatemala, Honduras, Nicaragua, and Panama. In South America, it is present in Colombia, Ecuador, French Guiana, Guyana, Peru, Suriname, Venezuela and the Brazilian states of Amazonas, Roraima, Rondônia, Bahia, Minas Gerais, Rio de Janeiro, and Mato Grosso do Sul (Fernández and Sendoya 2004; Branstetter and Sáenz 2012; Souza et al. 2018).

\section{(39) Paratrachymyrmex diversus (Mann, 1916)} Figure 10A, B

Material examined. COLOMBIA: Caquetá • 12 work-

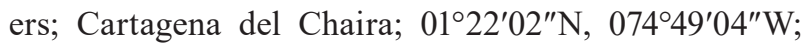
alt. 278 m; 28 Sep. 2016; UNAB members leg.; UNAB 4359; Caquetá - 12 workers; Florencia; 01³0'9.99"N, 075³6'23"W; alt. 250 m; 22 Sep. 2016; UNAB members leg.; UNAB 4359; Caquetá • 3 workers; Florencia; 01³0'9.99"N, 075³6'23"W; alt. 250 m; 21 Sep. 2016; F. Sernaleg.; UNAB 4359; Caquetá • 1 worker; Florencia;01 ${ }^{\circ}$ $37^{\prime} 11^{\prime \prime} \mathrm{N}$, 075'36'14"W; alt. 300 m; 31 Mar. 2016; F. Serna leg.; UNAB 4359; Caquetá • 1 worker; Florencia; $01^{\circ} 30^{\prime} 9.99^{\prime \prime} \mathrm{N}, 075^{\circ} 36^{\prime} 23^{\prime \prime} \mathrm{W}$; alt. $250 \mathrm{~m}$; 22 Sep. 2016; UNAB members leg.; UNAB 4359; Caquetá • 12 workers; Florencia; 01²5'34"N, 075³0'59"W; alt. 267 m; Sep. 2017; D. Mera-Rodríguez leg.; UNAB 4764; Caquetá - 12 workers; Florencia; 01²5'34"N, 075³0'59"W; alt. 267 m; Sep. 2017; D. Cubillos leg.; UNAB 4764.

Identification. Cephalic spines in lateral view robust, long and curved at apex; dorsum of propodeum with only one pair of, sometimes bifid, teeth; node of petiole in lateral view with oblique anterodorsal margin ending in a pair of robust posterodorsal spines; gaster with appressed hairs as well as short curved hairs; and hairs on legs appressed and curved with the apex reaching the tegument (unpublished key by Kempf).

Distribution. The distribution of $P$. diversus includes northern Brazil, Ecuador, and Venezuela (Kempf 1972; Brandão 1991; Fernández and Sendoya 2004; Ryder Wilkie et al. 2010). Our new distribution records for
Colombia (Fig. 1D) corroborates the northern South American distribution for this species.

\section{(40) Paratrachymyrmex irmgardae (Forel, 1912)}

Fig. 10C, D

Material examined. COLOMBIA: Cesar • 1 worker; Valledupar; $10^{\circ} 23^{\prime} 17^{\prime \prime} \mathrm{N}, 073^{\circ} 14^{\prime} 11^{\prime \prime} \mathrm{W}$; alt. 250-150 m; Jan. 2011; Y. Dominguez leg.; UNAB 765.

Identification. Cephalic denticle absent; mandibles striate but with no more than 12 striae between the lateral border and the basal teeth; and gaster with vestigial or no hair-tubercles (unpublished key by Kempf).

Distribution. Paratrachymyrmex irmgardae is only known from Colombia (Fig. 1D) (Kempf 1972).

\section{(41) Paratrachymyrmex phaleratus (Wheeler, 1925)} Fig. 10E, F

Material examined. COLOMBIA: Antioquia • 1 worker; Amalfi; 06 $55^{\prime} 00^{\prime \prime} \mathrm{N}, 075^{\circ} 04^{\prime} 00^{\prime \prime} \mathrm{W}$; alt. $1010 \mathrm{~m} ; 27$ Oct. 1997; F. Serna leg.; UNAB 5452.

Identification. Posterolateral cephalic corner with clearly distinct spine; mid-pronotal teeth present and separated from each other at the base; third pair of mesonotal spines distinct; and metasternal lamina dorsally rounded (unpublished key by Kempf).

Distribution. Paratrachymyrmex phaleratus was previously recorded in Guyana and Venezuela (Wheeler 1925; Mayhé-Nunes and Jaffé 1998), thus our new record for Colombia expands the geographic range of the species to the northwest Andean mountains (Fig. 1D).

\section{Discussion}

The importance of biological collections for studying biodiversity is underscored. In our study of the fungusgrowing ants housed at two of the main entomological collections in Colombia, we were able to identify a total of 41 species of non-leaf-cutting fungus-growing ants from 38 municipalities in Colombia, including distinct biogeographic regions such as the Orinoco, the Caribbean coast, the Pacific coast, the Andean mountains, and the Amazonian basin. Our study reveals the existence of five apparent new species and reports four new species records from Colombia. If we add to our results the species of non-leaf-cutting fungus-farming ants reported in Fernández and Serna (2019) but not encountered in this study, and the 12 species of leaf-cutting ants known to occur in the country (Fernández et al. 2015), we have a total of 85 fungus-growing ant species for Colombia. Colombia harbors approximately one-third of the known fungus-growing ant richness, which is higher than the neighboring countries of similar size and geographic location. For Venezuela, 54 fungus-growing ant species have been recorded (Fernández and Sendoya 2004; Guénard et al. 2017; Antweb 2020) and in Ecuador 51 fungus-growing ant species are known to occur (Fernández and Sendoya 2004; Salazar et al. 2015; Guénard et al. 


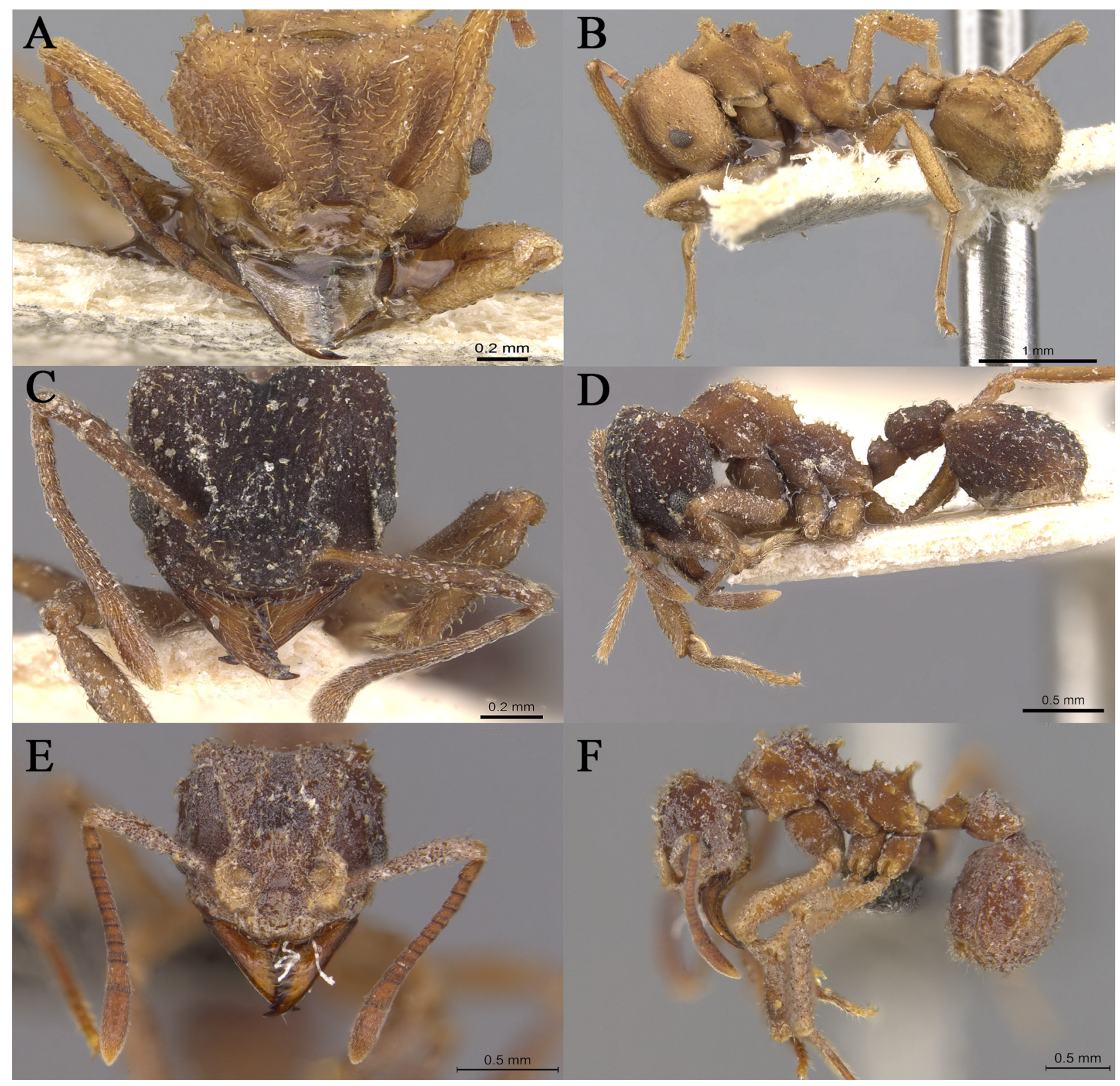

Figure 10. Paratrachymyrmex species encountered in Colombia. A, B. Paratrachymyrmex diversus, CASENT0901693. C, D. Paratrachymyrmex irmgardae, CASENT0909392. E, F. Paratrachymyrmex phaleratus, D. Mera-Rodríguez. Photographs taken by W. Erichson and obtained from the Antweb database (http://www.antweb.org).

2017). Panama is known to be home for at least 54 fungus-growing ant species (Fernández and Sendoya 2004; Antweb 2020) and in the Brazilian state of Amazonas, 66 species of fungus-growing ants have been recorded (Fernández and Sendoya 2004; Guénard et al. 2017).

In Colombia, the highest richness of non-leaf-cutting fungus-growing ant species was found in the departments of Caquetá (Amazonian region), Antioquia (Central Andean mountains), and Chocó (Pacific coast), harboring $60 \%(n=24$ species) of total attine species. The unequal species distribution observed across Colombian municipalities indicates a bias in sampling and/or curatorial efforts of researchers working in the two collections examined for this study. Our findings suggest that additional information of value regarding these ants in Colombia is likely to be found in other local collections, such as the Colección de Artrópodos Terrestres de la Amazonia Colombiana (CATAC) and the Instituto de Ciencias Naturales (ICN). Expanding both, sampling in the field and curation of museum holdings, is of paramount importance to gain a better understanding of insect diversity in Colombia.

Fungus-growing ants are a relatively small portion of the 1,166 ant species known in Colombia (Fernández and Serna 2019), but due to their significant economic impact, the leaf-cutting species have received much more attention from researchers than the other species (Vélez 1997; Chacón de Ulloa et al. 2006; Madrigal 2003; Della Lucia 2011; Della Lucia et al. 2014; Hölldobler and Wilson 2011; Montoya-Lerma et al. 2012; Fernández et al. 2015; Serna et al. 2019). The non-leaf-cutting attines remain mostly ignored and as a looming gap in our knowledge about the biology of the Atta genus-group. Future research needs to explore the richness, ecology, 
and biology of the non-leaf-cutting fungus-growing ants

We report several new distribution records for Colombia for species in the recently described genera Mycetomoellerius and Paratrachymyrmex. Based on a recent study by Solomon et al. (2019), the paraphyletic genus Trachymyrmex was divided into the three monophyletic genera Mycetomoellerius, Paratrachymyrmex, and Trachymyrmex. The genus Trachymyrmex sensu stricto contains nine described species, eight of which are restricted to northern Mexico and the southern United States and only a single species, $T$. saussurei (Forel, 1885), is known from Central America, ranging from Southern Mexico to Honduras (Rabeling et al. 2007; Sánchez-Peña et al. 2017). In contrast, species of Mycetomoellerius and Paratrachymyrmex are broadly distributed throughout Central and South America, and only a couple of Mycetomoellerius species, M. turrifex (Wheeler, 1903) and M. jamaicensis, occur in southern United States (MayhéNunes and Brandão 2007; Rabeling et al. 2007; Solomon et al. 2019).

Our results corroborate that Colombia harbors a significant diversity of non-leaf-cutting fungus-growing ant species, but also notes that for most of them, their biology, behavior, and ecology remain to be explored. Our study emphasizes the importance of integrating field and museum research for biodiversity exploration in the Neotropics. We hope our results will serve as a reference and an incentive for systematically exploring the diversity of fungus-growing ants in Colombia.

\section{Acknowledgements}

We thank both the UNAB entomological Museum at the Universidad Nacional de Colombia in Bogotá, and the MEFLG entomological Museum at the Universidad Nacional de Colombia in Medellín, as well as Arizona State University for providing the necessary tools for the identification of specimens and curatorial work. Also, we thank Erika Valentina Vergara, Jhon A. Quiroz, and Brandon Arredondo for supporting curatorial duties, as well as Sergio Rubiano for helping in assembling the geographic data. We thank the reviewers for their comments and suggestions in the manuscript.

Funding. This research was supported by the Universidad Nacional de Colombia, under the project "Curaduría y Sistematización del museo entomológico UNAB", Código: 44959, The Ministerio Colombiano de Ciencias (Minciencias) under the project "Reconocimiento de insectos de importancia agronomica asociados al agroecosistema Cacaotero de la Amazonia Colombiana", code 110171250625 CT FP44842-128-2016, and the US National Science Foundation (NSF DEB-1654829, NSF DEB-1927224, and NSF CAREER DEB-1943626).

\section{Authors Contributions}

DMR, FS designed and conducted the study. DMR, FS, JSC and CR identified the specimens. DMR and JSC took photographs. DMR wrote the manuscript. FS, JSC,
$\mathrm{JL}$, and CR improved the manuscript and participated in the discussion. DMR made the figures.

\section{References}

Achury R, Suárez AV (2018) Richness and composition of Grounddwelling Ants in tropical rainforest and surrounding landscapes in the Colombian Inter-Andean Valley. Neotropical Entomology 47: 731-741. https://doi.org/10.1007/s13744-017-0565-4

Adams RMM, Longino JT (2007) Nesting biology of the arboreal fungus-growing ant Cyphomyrmex cornutus and behavioral interactions with the social-parasitic ant Megalomyrmex mondabora. Insectes Sociaux 54: 136-143. https://doi.org/10.1007/s00040-0070922-0

Albuquerque EZ de (2014) Revisão taxonômica e analise filogenética do grupo rimosus de Cyphomyrmex (Hymenoptera, Formicidae, Attini). PhD dissertation, Universidade de São Paulo, São Paulo, 294 pp.

AntWeb (2020) Version 8.40.1. California Academy of Science. https:// www.antweb.org. Accessed on: 2020-7-21.

Bezděčková K, Bezděčka P, Machar I (2015) A checklist of the ants (Hymenoptera: Formicidae) of Peru. Zootaxa 4020 (1): 101-133. https://doi.org/10.11646/zootaxa.4020.1.4

Bolton B (2020) An online catalog of the ants of the world. AntCat. http://www.antcat.org. Accessed on: 2020-6-30.

Borgmeier T (1937) Formigas novas ou pouco conhecidas da América do Sul e Central, principalmente do Brasil (Hym. Formicidae). Archivos do Instituto de Biologia Vegetal 3 (2): 217-255.

Brandão CRF (1991) Adendos ao catálogo abreviado das formigas da região Neotropical (Hymenoptera: Formicidae). Revista Brasileira de Entomologia 35 (2): 319-412. https://doi.org/10.5281/zenodo. 24565

Branstetter MG, Sáenz L (2012) Las hormigas (Hymenoptera: Formicidae) de Guatemala. In: Cano EB, Schuster JC (Eds) Biodiversidad de Guatemala. Volumen 2. Universidad del Valle de Guatemala, Guatemala, 221-268.

Branstetter MG, Ješovnik A, Sosa-Calvo J, Lloyd MW, Faircloth BC, Brady SG, Schultz TR (2017) Dry habitats were crucibles of domestication in the evolution of agriculture in ants. Proceedings of the Royal Society B: Biological Sciences 284: 20170095. https:// doi.org/10.1098/rspb.2017.0095

Castro D, Fernández F, Meneses AD, Tocora MC, Sanchez S, PeñaVenegas CP (2018) A preliminary checklist of soil ants (Hymenoptera: Formicidae) of Colombian Amazon. Biodiversity Data Journal 6: e29278. https://doi.org/10.3897/BDJ.6.e29278

Chacón de Ulloa P, Jaramillo GI, Lozano MM (2006) Hormigas urbanas en el departamento del Valle del Cauca, Colombia. Revista de la Academia Colombiana de Ciencias Exactas, Físicas y Naturales 30 (116): 435-441.

Della Lucia TM (2011) Formigas cortadeiras da bioecologia ao manejo. Editora UFV, Viçosa, 421 pp.

Della Lucia TM, Gandra LC, Guedes RN (2014) Managing leaf-cutting ants: peculiarities, trends and challenges. Pest Management Science 70 (1): 14-23. https://doi.org/10.1002/ps.3660

Delsinne T, Serna FJ, Leponce M, Boudinot BE (2019) Glosario de morfología. In: Fernández F, Guerrero R, Delsinne T (Eds) Hormigas de Colombia. Universidad Nacional de Colombia, Bogotá, 387-457.

Demétrio MF, Silvestre R, Souza PR, Aoki C (2017) Inventário da fauna de formigas (Hymenoptera, Formicidae) no Mato Grosso do Sul, Brasil. Iheringia, Série Zoologia 107 (suppl.): e2017126. https://doi.org/10.1590/1678-4766e2017126

Deyrup M (2003) An updated list of Florida ants (Hymenoptera: Formicidae). The Florida Entomologist 86 (1): 43-48. https://doi. org/10.1653/0015-4040(2003)086[0043:aulofa]2.0.co;2

Deyrup M (2016) Ants of Florida. Identification and natural history. CRC Press, Boca Raton, 153 pp. 
Emery C (1894) Studi sulle formiche della fauna neotropica. Bullettino della Società Entomologica Italiana 26: 137-241.

Febvay G, Kermarrec A (1981) Morphologie et fonctionnement du filtre infrabuccal chez une attine Acromyrmex octospinosus (Reich) (Hymenoptera: Formicidae): role de la poche infrabuccale. International Journal of Insect Morphology and Embryology 10 (5-6) 441-449. https://doi.org/10.1016/0020-7322(81)90024-6.

Fernández F, Sendoya S (2004) Synonymic list of Neotropical ants (Hymenoptera: Formicidae). Biota Colombiana 5 (1): 3-105.

Fernández F, Serna F (2019) Subfamilia Myrmicinae. In: Fernández F, Guerrero R, Delsinne T (Eds) Hormigas de Colombia. Universidad Nacional de Colombia, Bogotá, 791-885.

Fernández F, Baena ML, Palacio EE (1996) Hormigas de Colombia V: el género Stenamma Westwood. Tacaya 5: 9-10.

Fernández F, Castro-Huertas V, Serna F (2015) Hormigas cortadoras de hojas en Colombia: Acromyrmex \& Atta (Hymenoptera: Formicidae). Universidad Nacional de Colombia, Bogotá, 354 pp.

Fichaux M, Béchade B, Donald J, Weyna A, Delabie JHC, Murienne J, Baraloto C, Orivel J (2019) Habitats shape taxonomic and functional composition of Neotropical ant assemblages. Oecologia 189: 501-513. https://doi.org/10.1007/s00442-019-04341-Z

Forel A (1912) Formicides néotropiques. Part I. Annales de la Société Entomologique de Belgique 56: 28-49.

Franco W, Feitosa RM (2018) First standardized inventory of ants (Hymenoptera: Formicidae) in the natural grasslands of Paraná: New records for Southern Brazil. Papéis Avulsos de Zoologia 58: e20185812. https://doi.org/10.11606/1807-0205/2018.58.12

Gallardo A (1915) Observaciones sobre algunas hormigas de la República Argentina. Anales del Museo Nacional de Historia Natural de Buenos Aires 27: 1-35.

García-Cárdenas R, Montoya-Lerma J, Armbrecht I (2018) Ant diversity under three coverages in a Neotropical coffee landscape. Revista de Biología Tropical 66 (4): 1373-1389. https://doi.org/10. 15517/rbt.v66i4.30610

Guénard B, Weiser MD, Gómez K, Narula N, Economo EP (2017) The Global Ant Biodiversity Informatics (GABI) database: a synthesis of ant species geographic distributions. Myrmecological News 24: 83-89.

Harada AY, Ketelhut SM (2009) Formigas da Reserva Ducke: um grupo ainda pouco estudado. In: Fonseca CRV, Magalhães C, Rafael JA, Franklin E (Eds) A fauna de artrópodes da Reserva Florestal Ducke: estado atual do conhecimento taxonômico e biológico. Editora INPA, Manaus, 231-248.

Hölldobler B, Wilson EO (1990) The ants. Harvard University Press, Cambridge, 732 pp.

Hölldobler B, Wilson EO (2011) Leaf-cutter ants. Civilization by instinct. W. W. Norton \& Company, New York, 160 pp.

Jaffé K, Lattke JE (1994) Ant fauna of the French and Venezuelan islands in the Caribbean. In: Williams DF (Ed) Exotic ants. Biology, impact, and control of introduced species. Westview Press, Boulder, 181-190.

Ješovnik A, Schultz TR (2017) Revision of the fungus-farming ant genus Sericomyrmex Mayr (Hymenoptera, Formicidae, Myrmicinae). ZooKeys 670: 1-109. https://doi.org/10.3897/zookeys.670. 11839

Kempf WW (1963) A review of the ant genus Mycocepurus Forel, 1893 (Hymenoptera: Formicidae). Studia Entomologica 6: 417-432.

Kempf WW (1965) A revision of the Neotropical fungus-growing ants of the genus Cyphomyrmex Mayr. Part II. Group of rimosus (Spinola) (Hym. Formicidae). Studia Entomologica 8: 161-200.

Kempf WW (1967) New ants from southeastern and central Brazil (Hymenoptera, Formicidae). Studia Entomologica 9: 121-128.

Kempf WW (1968) A new species of Cyphomyrmex from Colombia, with further remarks on the genus (Hymenoptera, Formicidae). Revista Brasileira de Biologia 28 (1): 35-41. https://doi.org/ 10.15468/EFWP0J

Kempf WW (1972) Catalogo abreviado das formigas da região neotropical (Hymenoptera: Formicidae). Studia Entomologica 15: 1-344.
Lattke JE (1997) Revisión del género Apterostigma Mayr: (Hymenoptera: Formicidae). Arquivos de Zoologia 34 (5): 121-221. https:// doi.org/10.11606/issn.2176-7793.v34i5p121-221

Li H, Sosa-Calvo J, Horn HA, Pupo MT, Clardy J, Rabeling C, Schultz TR, Currie CR (2018) Convergent evolution of complex structures for ant-bacterial defensive symbiosis in fungus-farming ants. Proceedings of the National Academy of Sciences 115 (42): 10720 10725. https://doi.org/10.1073/pnas.1809332115

Mackay W, Baena ML (1993) A new "Horned" Fungus Growing Ant Cyphomyrmex castagnei, from Colombia (Hymenoptera: Formicidae). Sociobiology 23 (1): 31-37.

Mackay WP, Maes JM, Fernández PR, Luna G (2004) The ants of North and Central America: the genus Mycocepurus (Hymenoptera: Formicidae). Journal of Insect Science 4 (27): 1-7. https://doi.org/10. 1093/jis/4.1.27

Madrigal A (2003) Las hormigas cortadoras Atta y Acromyrmex: biología, hábitos y ecología. In: Calderón LF (Ed.) Insectos forestales en Colombia. Biología, hábitos, ecología y manejo. Editorial Marín Vieco Ltda, Medellín, 369-396.

Martínez-Alava JO, Serna F (2015) Managing insect collections. Micropezidae (Diptera: Nerioidea) of the Entomological Museum UNAB. Agronomía Colombiana 33 (3): 339-347. https://doi.org/ 10.15446/agron.colomb.v33n3.52432

Mayhé-Nunes A, Jaffé K (1998) On the biogeography of Attini (Hymenoptera: Formicidae). Ecotropicos 11 (1): 45-54.

Mayhé-Nunes AJ, Brandão RF (2002) Revisionary studies on the Attine Ant genus Trachymyrmex Forel. Part 1: Definition of the genus and the Opulentus group (Hymenoptera: Formicidae). Sociobiology 40 (3): 667-698

Mayhé-Nunes AJ, Brandão RF (2005) Revisionary studies on the Attine Ant genus Trachymyrmex Forel. Part 2: The Iheringi group (Hymenoptera: Formicidae). Sociobiology 45 (2): 271-305.

Mayhé-Nunes AJ, Brandão CRF (2007) Revisionary studies on the attine ant genus Trachymyrmex Forel. Part 3: The Jamaicensis group (Hymenoptera: Formicidae). Zootaxa 1444 (1): 1-21. https://doi.org/10.11646/zootaxa.1444.1.1

Mehdiabadi NJ, Schultz TR (2010) Natural history and phylogeny of the fungus-farming ants (Hymenoptera: Formicidae: Myrmicinae: Attini). Myrmecological News 13: 37-55.

Miranda PN, Oliveira MA, Baccaro FB, Morato EF, Delabie JHC (2012) Check list of ground-dwelling ants (Hymenoptera: Formicidae) of the eastern Acre, Amazon, Brazil. Check List 8 (4): 722 730. https://doi.org/10.15560/8.4.722

Montoya-Lerma J, Giraldo-Echeverri C, Armbrecht I, Farji-Brener A, Calle Z (2012) Leaf-cutting ants revisited: towards rational management and control. International Journal of Pest Management 58 (3): 225-247. https://doi.org/10.1080/09670874.2012.663946

Nygaard S, Hu H, Li C, Schiøtt M, Chen Z, Yang Z, Xie Q, Ma C, Deng Y, Dikow RB, Rabeling C, Nash DR, Wcislo WT, Brady SG, Schultz TR, Zhang G, Boomsma JJ (2016) Reciprocal genomic evolution in the ant-fungus agricultural symbiosis. Nature Communications 7 (1): 12233. https://doi.org/10.1038/ncomms12233

QGIS (2020) QGIS Geographic Information System. Open Source Geospatial Foundation Project. http://qgis.org. Accessed on: 2020 9-10.

Rabeling C, Cover SP, Johnson RA, Mueller UG (2007) A review of the North American species of the fungus-gardening ant genus Trachymyrmex (Hymenoptera: Formicidae). Zootaxa 1664: 1-53.

Rabeling C, Gonzales O, Schultz TR, Bacci M Jr, Garcia MVB, Verhaagh M, Ishak HD, Mueller UG (2011) Cryptic sexual populations account for genetic diversity and ecological success in a widely distributed, asexual fungus-growing ant. Proceedings of the National Academy of Sciences 108 (30): 12366-12371. https:// doi.org/10.1073/pnas.1105467108

Rabeling C, Messer S, Lacau S, do Nascimento IC, Bacci M Jr, Delabie JHC (2019) Acromyrmex fowleri: a new inquiline social parasite species of leaf-cutting ants from South America, with a discussion of social parasite biogeography in the Neotropical region. 
Insectes Sociaux 66 (3): 435-451. https://doi.org/10.1007/s00040019-00705-z

Ryder Wilkie KT, Mertl AL, Traniello JFA (2010) Species diversity and distribution patterns of the ants of Amazonian Ecuador. PLOS ONE 5 (10): e13146. https://doi.org/10.1371/journal.pone.0013146

Salazar F, Reyes-Bueno F, Sanmartin D, Donoso DA (2015) Mapping continental Ecuadorian ant species. Sociobiology 6 (2):132-162. https://doi.org/10.13102/sociobiology.v62i2.132-162

Salinas PJ (2010) Catalogue of the ants of the Táchira State, Venezuela, with notes on their biodiversity, biogeography and ecology (Hymenoptera: Formicidae: Amblyioponinae, Ponerinae, Proceratiinae, Myrmicinae, Ecitoninae, Formicinae, Pseudomyrmecinae, Dolichoderinae). Boletín de la Sociedad Entomológica Aragonesa 47: 315-328.

Sánchez-Peña SR, Chacón-Cardosa MC, Canales-del-Castillo R, Ward L, Resendez-Pérez D (2017) A new species of Trachymyrmex (Hymenoptera, Formicidae) fungus-growing ant from the Sierra Madre Oriental of northeastern Mexico. ZooKeys 706: 73 94. https://doi.org/10.3897/zookeys.706.12539

Sanhudo CED (2011) Revisão taxonômica e relações filogenéticas do Grupo Strigatus de Cyphomyrmex Mayr, 1962 (Formicidae: Myrmicinae: Attini). PhD dissertation, Universidade Federal do Amazonas, Manaus, $111 \mathrm{pp}$

Santos R de J, Koch EBA, Leite CMP, Porto TJ, Delabie JHC (2017) An assessment of leaf-litter and epigaeic ants (Hymenoptera: Formicidae) living in different landscapes of the Atlantic Forest Biome in the State of Bahia, Brazil. Journal of Insect Biodiversity 5 (19): 1-19. https://doi.org/10.12976/jib/2017.5.19

Schultz TR, Brady SG (2008) Major evolutionary transitions in ant agriculture. Proceedings of the National Academy of Sciences of the United States of America 105 (14): 5435-5440. https://doi. org/10.1073/pnas.0711024105

Serna F, Mackay W (2010) A descriptive morphology of the Ant Genus Procryptocerus (Hymenoptera: Formicidae). Journal of Insect Science 10 (111): 1-36. https://doi.org/10.1673/031.010.11101

Serna F, Vergara-Navarro EV (2001) Claves para la identificacion de subfamilias y géneros de hormigas de Antioquia y Chocó, Colombia. Revista del Instituto de Ciencias Naturales y Ecología 7 (1): $5-41$.

Serna F, Bolton B, Mackay W (2011) On the morphology of Procryptocerus (Hymenoptera: Formicidae). Some comments and corrigenda. Zootaxa 2923 (1): 67-68. https://doi.org/10.11646/zootaxa. 2923.1 .5

Serna F, Mesa N, Vergara E, Quiroz J, Gaviria A (2017) Entomología Agrícola. In: Restrepo-Zea E, Sánchez C, Silva-Carrero G (Eds) Patrimonio de la Nación. Colección del Sesquicentenario. Universidad Nacional de Colombia, Medellín, 284-317.

Serna F, Mera-Rodríguez LD, Ramírez-Ossa K, Gaigl A (2019) Hormigas de mayor impacto en la agricultura colombiana. In: Fernández F, Guerrero RJ, Delsinne T (Eds) Hormigas de Colombia.
Universidad Nacional de Colombia, Bogotá, 1115-1149.

Snelling RR, Longino JT (1992) Revisionary notes on the fungusgrowing ants of the genus Cyphomyrmex, rimosus group (Hymenoptera: Formicidae: Attini). In: Quintero D, Aiello A (Eds) Insects of Panama and Mesoamerica: selected studies. Oxford University Press, Oxford, 479-494.

Solar RR de C, Barlow J, Andersen AN, Schoereder JH, Berenguer E, Ferreira JN, Gardner TA (2016) Biodiversity consequences of land-use change and forest disturbance in the Amazon: a multiscale assessment using ant communities. Biological Conservation 197: 98-107. https://doi.org/10.1016/j.biocon.2016.03.005

Solomon SE, Rabeling C, Sosa-Calvo J, Lopes CT, Rodrigues A, Vasconcelos HL, Bacci M Jr, Mueller UG, Schultz TR (2019) The molecular phylogenetics of Trachymyrmex Forel ants and their fungal cultivars provide insights into the origin and coevolutionary history of 'higher-attine' ant agriculture. Systematic Entomology 44 (4): 939-956. https://doi.org/10.1111/syen.12370

Sosa-Calvo J (2015) Systematics of the cryptic fungus-farming ant genus Myrmicocrypta Fr. Smith, with the description of a new genus and species of fungus-farming ants (Hymenoptera: Myrmicinae). PhD dissertation, University of Maryland, Maryland, 603 pp. https://doi.org/10.13016/M2VS6M

Sosa-Calvo J, Schultz TR, Ješovnik A, Dahan RA, Rabeling C (2018) Evolution, systematics, and natural history of a new genus of cryptobiotic fungus-growing ants. Systematic Entomology 43 (3): 549-567. https://doi.org/10.1111/syen.12289

Souza JLP, Baccaro FB, Pequeno PACL, Franklin E, Magnusson WE (2018) Effectiveness of genera as a higher-taxon substitute for species in ant biodiversity analyses is not affected by sampling technique. Biodiversity and Conservation 27 (13): 3425-3445. https:// doi.org/10.1007/s10531-018-1607-x

Vélez R (1997) Plagas agrícolas de impacto económico en Colombia: bionomía y manejo integrado. Universidad de Antioquia, Medellín, $482 \mathrm{pp}$.

Vergara-Navarro EV, Serna F (2013) A checklist of the ants (Hymenoptera: Formicidae) of the department of Antioquia, Colombia and new records for the country. Agronomía Colombiana 31 (3): 324-342.

Ward PS, Brady SG, Fisher BL, Schultz TR (2015) The evolution of myrmicine ants: phylogeny and biogeography of a hyperdiverse ant clade (Hymenoptera: Formicidae). Systematic Entomology 40 (1): 61-81. https://doi.org/10.1111/syen.12090

Weber NA (1938) The biology of the fungus-growing ants. Part IV. Additional new forms. Part V. The Attini of Bolivia. Revista de Entomología 9 (1-2): 154-206.

Weber NA (1972) The fungus-culturing behavior of Ants. American Zoologist 12 (3): 577-587. https://doi.org/10.1093/icb/12.3.577

Wheeler WM (1925) Neotropical ants in the collections of the Royal Museum of Stockholm. Part I. Arkiv för zoologi 17A (8): 1-55. 